\title{
Multiply Warped Products with a Semisymmetric Metric Connection
}

\author{
Yong Wang \\ School of Mathematics and Statistics, Northeast Normal University, Changchun, Jilin 130024, China \\ Correspondence should be addressed to Yong Wang; wangy581@nenu.edu.cn
}

Received 23 October 2013; Revised 3 January 2014; Accepted 15 January 2014; Published 28 April 2014

Academic Editor: Jaume Giné

Copyright ( $\odot 2014$ Yong Wang. This is an open access article distributed under the Creative Commons Attribution License, which permits unrestricted use, distribution, and reproduction in any medium, provided the original work is properly cited.

We study the Einstein multiply warped products with a semisymmetric metric connection and the multiply warped products with a semisymmetric metric connection with constant scalar curvature, and we apply our results to generalized Robertson-Walker space-times with a semisymmetric metric connection and generalized Kasner space-times with a semisymmetric metric connection and find some new examples of Einstein manifolds with a semisymmetric metric connection and manifolds with constant scalar curvature with a semisymmetric metric connection.

\section{Introduction}

The (singly) warped product $B \times{ }_{b} F$ of two pseudoRiemannian manifolds $\left(B, g_{B}\right)$ and $\left(F, g_{F}\right)$ with a smooth function $b: B \rightarrow(0, \infty)$ is the product manifold $B \times F$ with the metric tensor $g=g_{B} \oplus b^{2} g_{F}$. Here, $\left(B, g_{B}\right)$ is called the base manifold and $\left(F, g_{F}\right)$ is called the fiber manifold and $b$ is called the warping function. Generalized RobertsonWalker space-times and standard static space-times are two well-known warped product spaces. The concept of warped products was first introduced by Bishop and O'Neill (see [1]) to construct examples of Riemannian manifolds with negative curvature. In Riemannian geometry, warped product manifolds and their generic forms have been used to construct new examples with interesting curvature properties since then. In [2], Dobarro and Dozo had studied from the viewpoint of partial differential equations and variational methods the problem of showing when a Riemannian metric of constant scalar curvature can be produced on product manifolds by a warped product construction. In [3], Ehrlich et al. got explicit solutions to warping function to have a constant scalar curvature for generalized Robertson-Walker space-times. In [4], explicit solutions were also obtained for the warping function to make the space-time as Einstein when the fiber is also Einstein.

One can generalize singly warped products to multiply warped products. Briefly, a multiply warped product $(M, g)$ is a product manifold of form $M=B \times_{b_{1}} F_{1} \times{ }_{b_{2}} F_{2} \cdots \times_{b_{m}} F_{m}$ with the metric $g=g_{B} \oplus b_{1}^{2} g_{F_{1}} \oplus b_{2}^{2} g_{F_{2}} \cdots \oplus b_{m}^{2} g_{F_{m}}$, where for each $i \in\{1, \ldots, m\}, b_{i}: B \stackrel{\rightarrow}{\rightarrow}(0, \infty)$ is smooth and $\left(F_{i}, g_{F_{i}}\right)$ is a pseudo-Riemannian manifold. In particular, when $B=(c, d)$ with the negative definite metric $g_{B}=-d t^{2}$ and $\left(F_{i}, g_{F_{i}}\right)$ is a Riemannian manifold, we call $M$ the multiply generalized Robertson-Walker space-time. In [5], Dobarro and Ünal studied Ricci-flat and Einstein-Lorentzian multiply warped products and considered the case of having constant scalar curvature for multiply warped products and applied their results to generalized Kasner space-times.

Singly warped products have a natural generalization. A twisted product $(M, g)$ is a product manifold of form $M=B \times{ }_{b} F$, with a smooth function $b: B \times F \rightarrow$ $(0, \infty)$, and the metric tensor $g=g_{B} \oplus b^{2} g_{F}$. In [6], they showed that mixed Ricci-flat twisted products could be expressed as warped products. As a consequence, any Einstein twisted products are warped products. In this paper, we define the multiply twisted products as generalizations of multiply warped products and twisted products. A multiply twisted product $(M, g)$ is a product manifold of form $M=$ $B \times_{b_{1}} F_{1} \times b_{b_{2}} F_{2} \cdots \times_{b_{m}} F_{m}$ with the metric $g=g_{B} \oplus b_{1}^{2} g_{F_{1}} \oplus$ $b_{2}^{2} g_{F_{2}} \cdots \oplus b_{m}^{2} g_{F_{m}}$, where for each $i \in\{1, \ldots, m\}, b_{i}: B \times F_{i} \rightarrow$ $(0, \infty)$ is smooth.

The definition of a semisymmetric metric connection was given by Hayden in [7]. In 1970, Yano [8] considered 
a semisymmetric metric connection and studied some of its properties. He proved that a Riemannian manifold admitting the semisymmetric metric connection has vanishing curvature tensor if and only if it is conformally flat. Motivated by the Yano result, in [9], Sular and Özgür studied warped product manifolds with a semisymmetric metric connection; they computed curvature of semisymmetric metric connection and considered Einstein warped product manifolds with a semisymmetric metric connection. In this paper, we considered multiply twisted products with a semisymmetric metric connection and computed the curvature of a semisymmetric metric connection. We showed that mixed Ricci-flat multiply twisted products with a semisymmetric metric connection can be expressed as multiply warped products which generalizes the result in [6]. We also studied the Einstein multiply warped products with a semisymmetric metric connection and multiply warped products with a semisymmetric metric connection with constant scalar curvature; we applied our results to generalized Robertson-Walker space-times with a semisymmetric metric connection and generalized Kasner space-times with a semisymmetric metric connection and we found some new examples of Einstein affine manifolds and affine manifolds with constant scalar curvature. We also classified generalized Einstein Robertson-Walker space-times with a semisymmetric metric connection and generalized Einstein Kasner space-times with a semisymmetric metric connection.

Semisymmetric metric connections have some physical applications. In $[10,11]$, they considered orthogonal connections with arbitrary torsion on compact Riemannian manifolds. For the induced Dirac operators, twisted Dirac operators, and Dirac operators of Chamseddine-Connes type, they computed the spectral action. In addition to the Einstein-Hilbert action and the bosonic part of the standard model Lagrangian, they found the Holst term from loop quantum gravity, a coupling of the Holst term to the scalar curvature, and a prediction for the value of the BarberoImmirzi parameter. For connections whose torsion is not zero, they showed that the Holst action can be recovered from the heat asymptotics for the natural Dirac operator acting on spinor fields. For the physical consequences of the use of torsion connections in Lorentzian geometry, we refer to the classical review [12] and the more recent overview [13] and references therein.

This paper is arranged as follows. In Section 2, we compute curvature of multiply twisted products with a semisymmetric metric connection. In Section 3, we study the special multiply warped products with a semisymmetric metric connection. In Section 4, we study the generalized Robertson-Walker space-times with a semisymmetric metric connection. In Section 5, we consider the generalized Kasner space-times with a semisymmetric metric connection.

\section{Preliminaries}

Let $M$ be a Riemannian manifold with Riemannian metric g. A linear connection $\bar{\nabla}$ on a Riemannian manifold $M$ is called a semisymmetric connection if the torsion tensor $T$ of the connection $\bar{\nabla}$

$$
T(X, Y)=\bar{\nabla}_{X} Y-\bar{\nabla}_{Y} X-[X, Y]
$$

satisfies

$$
T(X, Y)=\pi(Y) X-\pi(X) Y,
$$

where $\pi$ is a one form associated with the vector field $P$ on $M$ defined by $\pi(X)=g(X \cdot P) . \bar{\nabla}$ is called a semisymmetric metric connection if it satisfies $\bar{\nabla} g=0$. If $\nabla$ is the Levi-Civita connection of $M$, the semisymmetric metric connection $\bar{\nabla}$ is given by

$$
\bar{\nabla}_{X} Y=\nabla_{X} Y+\pi(Y) X-g(X, Y) P,
$$

(see [8]). Let $R$ and $\bar{R}$ be the curvature tensors of $\nabla$ and $\bar{\nabla}$, respectively. Then $R$ and $\bar{R}$ are related by

$$
\begin{aligned}
\bar{R}(X, Y) Z= & R(X, Y) Z+g\left(Z, \nabla_{X} P\right) Y-g\left(Z, \nabla_{Y} P\right) X \\
& +g(X, Z) \nabla_{Y} P-g(Y, Z) \nabla_{X} P \\
& +\pi(P)[g(X, Z) Y-g(Y, Z) X] \\
& +[g(Y, Z) \pi(X)-g(X, Z) \pi(Y)] P \\
& +\pi(Z)[\pi(Y) X-\pi(X) Y],
\end{aligned}
$$

for any vector fields $X, Y, Z$ on $M$ [8]. By (3) and Proposition 2, we have a multiply twisted product $(M, g)$ which is a product manifold of form $M=B \times_{b_{1}} F_{1} \times_{b_{2}} F_{2} \cdots \times_{b_{m}} F_{m}$ with the metric $g=g_{B} \oplus b_{1}^{2} g_{F_{1}} \oplus b_{2}^{2} g_{F_{2}} \cdots \oplus b_{m}^{2} g_{F_{m}}$, where for each $i \in\{1, \ldots, m\}, b_{i}: B \times F_{i} \rightarrow(0, \infty)$ is smooth.

Here, $\left(B, g_{B}\right)$ is called the base manifold and $\left(F_{i}, g_{F_{i}}\right)$ is called the fiber manifold and $b_{i}$ is called the twisted function. Obviously, twisted products and multiply warped products are the special cases of multiply twisted products.

Proposition 1 (compare with [5, Proposition 2.2]). Let $M=$ $B \times{ }_{b_{1}} F_{1} \times_{b_{2}} F_{2} \cdots \times_{b_{m}} F_{m}$ be a multiply twisted product and let $X, Y \in \Gamma(T B)$ and $U \in \Gamma\left(T F_{i}\right), W \in \Gamma\left(T F_{j}\right)$ and $P \in \Gamma(T B)$. Then

(1) $\bar{\nabla}_{X} Y=\bar{\nabla}_{X}^{B} Y$;

(2) $\bar{\nabla}_{X} U=\left(X\left(b_{i}\right) / b_{i}\right) U$;

(3) $\bar{\nabla}_{U} X=\left[X\left(b_{i}\right) / b_{i}+\pi(X)\right] U$;

(4) $\bar{\nabla}_{U} W=0$ if $i \neq j$;

(5) $\bar{\nabla}_{U} W=U\left(\ln b_{i}\right) W+W\left(\ln b_{i}\right) U-\left(g_{F_{i}}(U, W) /\right.$ $\left.b_{i}\right) \operatorname{grad}_{F_{i}} b_{i}-b_{i} g_{F_{i}}(U, W) \operatorname{grad}_{B} b_{i}+\nabla_{U}^{F_{i}} W-g(U, W) P$ if $i=j$,

where $\bar{\nabla}^{B}$ denotes the semisymmetric metric connection on $B$ and $\operatorname{grad}_{B} b_{i}, \operatorname{grad}_{F_{i}} b_{i}$ denote the gradient vector fields on $B$ and $F_{i}$, respectively.

Proof. Similar to Proposition 2.2 in [5] and Proposition 1 in [6], we have 
(1) $\nabla_{X} Y=\nabla_{X}^{B} Y$;

(2) $\nabla_{X} U=\nabla_{U} X=\left(X\left(b_{i}\right) / b_{i}\right) U$;

(3) $\nabla_{U} W=0$ if $i \neq j$;

(4) $\nabla_{U} W=U\left(\ln b_{i}\right) W+W\left(\ln b_{i}\right) U-\left(g_{F_{i}}(U, W) /\right.$ $\left.b_{i}\right) \operatorname{grad}_{F_{i}} b_{i}-b_{i} g_{F_{i}}(U, W) \operatorname{grad}_{B} b_{i}+\nabla_{U}^{F_{i}} W$ if $i=j$.

So by (3), we get Proposition 1.

Similar to the proof of Proposition 1, we get the following.

Proposition 2 (compare with [5, Proposition 2.2]). Let $M=$ $B \times_{b} F_{1} \times_{b} F_{2} \cdots \times_{b} F_{m}$ be a multiply twisted product and let $X, Y \in \Gamma(T B)$ and $U \in \Gamma\left(T F_{i}\right), W \in \Gamma\left(T F_{j}\right)$ and $P \in \Gamma\left(T F_{k}\right)$ for a fixed $k$. Then

(1) $\bar{\nabla}_{X} Y=\nabla_{X}^{B} Y-g(X, Y) P$;

(2) $\bar{\nabla}_{X} U=\left(X\left(b_{i}\right) / b_{i}\right) U+g(P, U) X$;

(3) $\bar{\nabla}_{U} X=\left(X\left(b_{i}\right) / b_{i}\right) U$;

(4) $\bar{\nabla}_{U} W=g(W, P) U$ if $i \neq j$;

(5) $\bar{\nabla}_{U} W=U\left(\ln b_{i}\right) W+W\left(\ln b_{i}\right) U-\left(g_{F_{i}}(U, W) /\right.$ $\left.b_{i}\right) \operatorname{grad}_{F_{i}} b_{i}-b_{i} g_{F_{i}}(U, W) \operatorname{grad}_{B} b_{i}+\nabla_{U}^{F_{i}} W+\pi(W) U-$ $g(U, W) P$ if $i=j$.

Remark 3. When for each $i \in\{1, \ldots, m\}, b_{i}: B \rightarrow(0, \infty)$ is smooth and $P=0$, we get Proposition 2.2 in [5] by Propositions 1 and 2.

Define the curvature, Ricci curvature, and scalar curvature as follows:

$$
\begin{gathered}
R(X, Y) Z=\nabla_{X} \nabla_{Y}-\nabla_{Y} \nabla_{X}-\nabla_{[X, Y]}, \\
\operatorname{Ric}(X, Y)=\sum_{k} \varepsilon_{k}\left\langle R\left(X, E_{k}\right) Y, E_{k}\right\rangle, \\
S=\sum_{k} \varepsilon_{k} \operatorname{Ric}\left(E_{k}, E_{k}\right),
\end{gathered}
$$

where $E_{k}$ is an orthonormal base of $M$ with $\left\langle E_{k}, E_{k}\right\rangle=\varepsilon_{k}$. The Hessian of $f$ is defined by $H^{f}(X, Y)=X Y f-\left(\nabla_{X} Y\right) f$.

Proposition 4 (compare with [5, Proposition 2.4]). Let $M=$ $B \times_{b_{1}} F_{1} \times_{b_{2}} F_{2} \cdots \times_{b_{m}} F_{m}$ be a multiply twisted product and let $X, Y, Z \in \Gamma(T B)$ and $V \in \Gamma\left(T F_{i}\right), W \in \Gamma\left(T F_{j}\right), U \in \Gamma\left(T F_{k}\right)$, and $P \in \Gamma(T B)$. Then

(1) $\bar{R}(X, Y) Z=\bar{R}^{B}(X, Y) Z$;

(2) $\bar{R}(V, X) Y=-\left[\left(H_{B}^{b_{i}}(X, Y) / b_{i}\right)+\left(P\left(b_{i}\right) / b_{i}\right) g(X, Y)+\right.$ $\left.\pi(P) g(X, Y)+g\left(Y, \nabla_{X} P\right)-\pi(X) \pi(Y)\right] V ;$

(3) $\bar{R}(X, V) W=\bar{R}(V, W) X=\bar{R}(V, X) W=0$ if $i \neq j$;

(4) $\bar{R}(X, Y) V=0$;

(5) $\bar{R}(V, W) X=V X\left(\ln b_{i}\right) W-W X\left(\ln b_{i}\right) V$ if $i=j$;

(6) $\bar{R}(V, W) U=0$ if $i=j \neq k$ or $i \neq j \neq k$;

(7) $\bar{R}(U, V) W=-g(V, W)\left(g_{B}\left(\operatorname{grad}_{B} \mathrm{~b}_{\mathrm{i}}, \operatorname{grad}_{B} \mathrm{~b}_{\mathrm{k}}\right) / \mathrm{b}_{\mathrm{i}} \mathrm{b}_{\mathrm{k}}\right) \mathrm{U}-$ $\mathrm{g}(\mathrm{V}, \mathrm{W})\left(\left(\mathrm{P}\left(\mathrm{b}_{\mathrm{i}}\right) / \mathrm{b}_{\mathrm{i}}\right)+\left(\mathrm{P}\left(\mathrm{b}_{\mathrm{k}}\right) / \mathrm{b}_{\mathrm{k}}\right)\right) \mathrm{U}-\pi(\mathrm{P}) \mathrm{g}(\mathrm{V}, \mathrm{W}) \mathrm{U}$, if $i=j \neq k$;
(8) $\bar{R}(X, V) W=\left[W X\left(\ln b_{i}\right)\right] V-g(W, V) \cdot\left[\left(\nabla_{X}^{B}\left(\operatorname{grad}_{B} b_{i}\right) /\right.\right.$ $\left.b_{i}\right)+\left(\operatorname{grad}_{F_{i}}\left(X \ln b_{i}\right) / b_{i}^{2}\right)+\left(P\left(b_{i}\right) / b_{i}\right) X+\nabla_{X} P+\pi(P) X-$ $\pi(X) P]$ if $i=j$;

(9) $\bar{R}(U, V) W=g(U, W) \operatorname{grad}_{B}\left(V\left(\ln b_{i}\right)\right)-g(V$, $W) \operatorname{grad}_{B}\left(U\left(\ln b_{i}\right)\right)+R^{F_{i}}(U, V) W-\left(\left(\left|\operatorname{grad}_{B} b_{i}\right|_{B}^{2} / b_{i}^{2}\right)+\right.$ $\left.2\left(P\left(b_{i}\right) / b_{i}\right)+\pi(P)\right)(g(V, W) U-g(U, W) V)$ if $i=j=k$.

Proof. Similar to Proposition 2.4 in [5], we have

(1) $R(X, Y) Z=R^{B}(X, Y) Z$;

(2) $R(V, X) Y=-\left(H_{B}^{b_{i}}(X, Y) / b_{i}\right) V$;

(3) $R(X, V) W=R(V, W) X=R(V, X) W=0$ if $i \neq j$;

(4) $R(X, Y) V=0$;

(5) $R(V, W) X=V X\left(\ln b_{i}\right) W-W X\left(\ln b_{i}\right) V$ if $i=j$;

(6) $R(V, W) U=0$ if $i=j \neq k$ or $i \neq j \neq k$;

(7) $R(U, V) W=-g(V, W)\left(g_{B}\left(\operatorname{grad}_{B} b_{i}, \operatorname{grad}_{B} b_{k}\right) / b_{i} b_{k}\right) U$, if $i=j \neq k$;

(8) $R(X, V) W=-\left(g(V, W) / b_{i}\right) \nabla_{X}^{B}\left(\operatorname{grad}_{B} b_{i}\right)+$ $\left[W X\left(\ln b_{i}\right)\right] V-g_{F_{i}}(W, V) \operatorname{grad}_{F_{i}}\left(X \ln b_{i}\right)$ if $i=j$;

(9) $R(V, W) U=g(V, U) \operatorname{grad}_{B}\left(W\left(\ln b_{i}\right)\right)-g(W$, $U) \operatorname{grad}_{B}\left(V\left(\ln b_{i}\right)\right)+R^{F_{i}}(V, W) U-\left(\left|\operatorname{grad}_{B} b_{i}\right|_{B}^{2} / b_{i}^{2}\right)$ $(g(W, U) V-g(V, U) W)$ if $i=j=k$.

Then by (4), we get Proposition 4 .

Similarly we may get the following.

Proposition 5 (compare with [5, Proposition 2.4]). Let $M=$ $B \times{ }_{b_{1}} F_{1} \times_{b_{2}} F_{2} \cdots \times_{b_{m}} F_{m}$ be a multiply twisted product and let $X, Y, Z \in \Gamma(T B)$ and $V \in \Gamma\left(T F_{i}\right), W \in \Gamma\left(T F_{j}\right), U \in \Gamma\left(T F_{k}\right)$, and $P \in \Gamma\left(T F_{l}\right)$ for a fixed $l$. Then

(1) $\bar{R}(X, Y) Z=R^{B}(X, Y) Z+\left[g(X, Z)\left(Y b_{l} / b_{l}\right)-\right.$ $\left.g(Y, Z)\left(X b_{l} / b_{l}\right)\right] P+\pi(P)[g(X, Z) Y-g(Y, Z) X] ;$

(2) $\bar{R}(V, X) Y=-\left(H_{B}^{b_{i}}(X, Y) / b_{i}\right) V-\pi(P) g(X, Y) V$ if $i \neq l$;

(3) $\bar{R}(V, X) Y=-\left(H_{B}^{b_{i}}(X, Y) / b_{i}\right) V-\pi(V)\left(Y\left(b_{i}\right) / b_{i}\right) X-$ $g(X, Y) \nabla_{V} P-g(X, Y)[\pi(P) V-\pi(V) P]$ if $i=l ;$

(4) $\bar{R}(X, V) W=\left(X\left(b_{l}\right) / b_{l}\right) \pi(W) V$ if $i \neq j$;

(5) $\bar{R}(V, W) X=-\delta_{i}^{l}\left(\pi(V) / b_{i}\right) X\left(b_{i}\right) W+\delta_{j}^{l}(\pi(W) /$ $\left.b_{j}\right) X\left(b_{j}\right) V$ if $i \neq j$;

(6) $\bar{R}(X, Y) V=\pi(V)\left[\left(X\left(b_{l}\right) / b_{l}\right) Y-\left(Y\left(b_{l}\right) / b_{l}\right) X\right]$;

(7) $\bar{R}(V, W) X=V X\left(\ln b_{i}\right) W-W X\left(\ln b_{i}\right) V-\delta_{i}^{l}\left(X\left(b_{i}\right) /\right.$ $\left.b_{i}\right)[\pi(V) W-\pi(W) V]$ if $i=j$;

(8) $\bar{R}(V, W) U=0$ if $i=j \neq k$ or $i \neq j \neq k$;

(9) $\bar{R}(U, V) W=-g(V, W)\left(g_{B}\left(\operatorname{grad}_{B} b_{i}, \operatorname{grad}_{B} b_{k}\right) / b_{i} b_{k}\right) U-$ $g\left(W, \nabla_{V} P\right) U-g(V, W) \nabla_{U} P-\pi(P) g(V, W) U+$ $g(V, W) \pi(U) P+\pi(W)[\pi(V) U-\pi(U) V]$, if $i=j \neq k ;$

(10) $\bar{R}(X, V) W=\left[W X\left(\ln b_{i}\right)\right] V-g(W, V)\left(\nabla_{X}^{B}\left(\operatorname{grad}_{B} b_{i}\right) /\right.$ $\left.b_{i}\right)-\operatorname{grad}_{F_{i}}\left(X \ln b_{i}\right) g_{F_{i}}(W, V)+\left(X\left(b_{l}\right) / b_{l}\right) \pi(W) V-$ $g\left(W, \nabla_{V} P\right) X-g(V, W)\left(X\left(b_{l}\right) / b_{l}\right) P-g(V, W) \pi(P) X+$ $\pi(V) \pi(W) X$ if $i=j$; 
(11) $\bar{R}(U, V) W=g(U, W) \operatorname{grad}_{B}\left(V\left(\ln b_{i}\right)\right)-g(V$, $W) \operatorname{grad}_{B}\left(U\left(\ln b_{i}\right)\right)+R^{F_{i}}(U, V) W-\left(\left|\operatorname{grad}_{B} b_{i}\right|_{B}^{2} /\right.$ $\left.b_{i}^{2}\right)(g(V, W) U-g(U, W) V)+\pi(P)[g(U, W) V-g(V$, $W) U]$ if $i=j=k \neq l$;

(12) $\bar{R}(U, V) W=g(U, W) \operatorname{grad}_{B}\left(V\left(\ln b_{i}\right)\right)-g(V$, $W) \operatorname{grad}_{B}\left(U\left(\ln b_{i}\right)\right)+R^{F_{i}}(U, V) W-\left(\left|\operatorname{grad}_{B} b_{i}\right|_{B}^{2} / b_{i}^{2}\right)$ $(g(V, W) U-g(U, W) V)+g\left(W, \nabla_{U} P\right) V-g\left(W, \nabla_{V} P\right) U+$ $g(U, W) \nabla_{V} P-g(V, W) \nabla_{U} P+\pi(P)[g(U, W) V-$ $g(V, W) U]+[g(V, W) \pi(U)-g(U, W) \pi(V)] P+$ $\pi(W)[\pi(V) U-\pi(U) V]$ if $i=j=k=l$, where $\delta_{i}^{l}$ denotes the Kronecker symbol.

Remark 6. When for each $i \in\{1, \ldots, m\}, b_{i}: B \rightarrow(0, \infty)$ is smooth and $P=0$, we get Proposition 2.4 in [5] by Propositions 4 and 5.

By Propositions 4 and 5 and the definition of the Ricci curvature tensor, we have the following.

Proposition 7 (compare with [5, Proposition 2.5]). Let $M=$ $B \times{ }_{b_{1}} F_{1} \times \times_{b_{2}} F_{2} \cdots \times_{b_{m}} F_{m}$ be a multiply twisted product and let $X, Y, Z \in \Gamma(T B)$ and $V \in \Gamma\left(T F_{i}\right), W \in \Gamma\left(T F_{j}\right)$, and $P \in \Gamma(T B)$. Then

(1) $\overline{\operatorname{Ric}}(X, Y)=\overline{\operatorname{Ric}}^{B}(X, Y)+\sum_{i=1}^{m} l_{i}\left[\left(H_{B}^{b_{i}}(X, Y) / b_{i}\right)+\right.$ $\left(P\left(b_{i}\right) / b_{i}\right) g(X, Y)+\pi(P) g(X, Y)+g\left(Y, \nabla_{X} P\right)-$ $\pi(X) \pi(Y)]$

(2) $\overline{\operatorname{Ric}}(X, V)=\overline{\operatorname{Ric}}(V, X)=\left(l_{i}-1\right)\left[V X\left(\ln b_{i}\right)\right]$;

(3) $\overline{\operatorname{Ric}}(V, W)=0$ if $i \neq j$;

(4) $\overline{\operatorname{Ric}}(V, W)=\operatorname{Ric}^{F_{i}}(V, W)+\left[\left(\Delta_{B} b_{i} / b_{i}\right)+\left(l_{i}-\right.\right.$ 1) $\left(\left|\operatorname{grad}_{B} b_{i}\right|_{B}^{2} / b_{i}^{2}\right)+\sum_{j \neq i} l_{j}\left(g_{B}\left(\operatorname{grad}_{B} b_{i}, \operatorname{grad}_{B} b_{j}\right) /\right.$ $\left.b_{i} b_{j}\right)+(\bar{n}-2) \pi(P)+\sum_{k=1}^{n} \varepsilon_{k}\left\langle\nabla_{E_{k}} P, E_{k}\right\rangle+$ $\left.\sum_{\substack{j \neq i \\ i=j}} l_{j}\left(P b_{j} / b_{j}\right)+\left(\bar{n}+l_{i}-2\right)\left(P b_{i} / b_{i}\right)\right] g(V, W)$ if

where $E_{k}, 1 \leq k \leq n$ is an orthonormal base of $B$ with $\varepsilon_{k}=$ $g\left(E_{k}, E_{k}\right)$ and $\operatorname{dim} B=n, \operatorname{dim} M=\bar{n}$.

By (2) and (3) in Proposition 7, similar to the proof of Theorem 1 in [6], we have the following.

Corollary 8. Let $M=B \times_{b_{1}} F_{1} \times_{b_{2}} F_{2} \cdots \times_{b_{m}} F_{m}$ be a multiply twisted product and $\operatorname{dim} F_{i}>1$ and $P \in \Gamma(T B)$; then $(M, \bar{\nabla})$ is mixed Ricci-flat if and only if $M$ can be expressed as a multiply warped product. In particular, if $(M, \bar{\nabla})$ is Einstein, then $M$ can be expressed as a multiply warped product.

Similar to Proposition 7, we have the following.

Proposition 9 (compare with [5, Proposition 2.5]). Let $M=$ $B \times_{b_{1}} F_{1} \times_{b_{2}} F_{2} \cdots \times_{b_{m}} F_{m}$ be a multiply twisted product and let $X, Y, Z \in \Gamma(T B)$ and $V \in \Gamma\left(T F_{i}\right), W \in \Gamma\left(T F_{j}\right)$, and $P \in \Gamma\left(T F_{r}\right)$ for a fixed $r$. Then

(1) $\overline{\operatorname{Ric}}(X, Y)=\operatorname{Ric}^{B}(X, Y)+\sum_{i=1}^{m} l_{i}\left(H_{B}^{b_{i}}(X, Y) / b_{i}\right)+$ $g(X, Y) \pi(P)(\bar{n}-2)+g(X, Y) \sum_{q=1}^{l_{r}} \varepsilon_{q} g\left(\nabla_{E_{q}} P, E_{q}\right)$, where $E_{q}$ for $1 \leq q \leq l_{r}$ is an orthonormal basis of $T F_{r}$;
(2) $\overline{\operatorname{Ric}}(X, V)=\left(l_{i}-1\right)\left[V X\left(\ln b_{i}\right)\right]+(\bar{n}-2)\left(X\left(b_{r}\right) / b_{r}\right) \pi(V)$;

(3) $\overline{\operatorname{Ric}}(V, X)=\left(l_{i}-1\right)\left[V X\left(\ln b_{i}\right)\right]+(2-\bar{n})\left(X\left(b_{r}\right) / b_{r}\right) \pi(V)$;

(4) $\overline{\operatorname{Ric}}(V, W)=0$ if $i \neq j$;

(5) $\overline{\operatorname{Ric}}(V, W)=\operatorname{Ric}^{\mathrm{F}_{\mathrm{i}}}(\mathrm{V}, \mathrm{W})+\mathrm{g}(\mathrm{V}, \mathrm{W})\left[\left(\Delta_{\mathrm{B}} \mathrm{b}_{\mathrm{i}} /\right.\right.$ $\left.b_{\mathrm{i}}\right)+\left(l_{i}-1\right)\left(\left|\operatorname{grad}_{B} b_{i}\right|_{B}^{2} / b_{i}^{2}\right)+\sum_{k, k \neq i} l_{k}\left(g_{B}\left(\operatorname{grad}_{B} b_{i}\right.\right.$, $\left.\left.\left.\operatorname{grad}_{B} b_{k}\right) / b_{i} b_{k}\right)+(\bar{n}-2) \pi(P)\right]+(\bar{n}-2) g\left(W, \nabla_{V} P\right)+$ $(2-\bar{n}) \pi(V) \pi(W)+g(V, W) \operatorname{div}_{F_{r}} P$ if $i=j$.

Remark 10. When for each $i \in\{1, \ldots, m\}, b_{i}: B \rightarrow(0, \infty)$ is smooth and $P=0$, we get Proposition 2.5 in [5] by Propositions 7 and 9.

Corollary 11. Let $M=B \times_{b_{1}} F_{1} \times_{b_{2}} F_{2} \cdots \times_{b_{m}} F_{m}$ be a multiply twisted product and $\operatorname{dim} F_{i}>1$ and $P \in \Gamma\left(T F_{r}\right)$; then $(M, \bar{\nabla})$ is mixed Ricci-flat if and only if $M$ can be expressed as a multiply warped product and $b_{r}$ is only dependent on $F_{r}$. In particular, if $(M, \bar{\nabla})$ is Einstein, then $M$ can be expressed as a multiply warped product.

Proof. By (2) and (3) in Proposition 9, we have that $(M, \bar{\nabla})$ is mixed Ricci-flat if and only if $\left[V X\left(\ln b_{i}\right)\right]=$ $0,\left(X\left(b_{r}\right) / b_{r}\right) \pi(V)=0$. By $\left[V X\left(\ln b_{i}\right)\right]=0$, similar to the proof of Corollary 8 , we get that $M$ can be expressed as a multiply warped product. When $i \neq r, \pi(V)=0$. When $i=r$, by $\left(X\left(b_{r}\right) / b_{r}\right) \pi(V)=0$, then $b_{r}$ depends only on $M_{r}$.

By Proposition 7 and the definition of the scalar curvature, we have the following.

Proposition 12 (compare with [5, Proposition 2.6]). Let $M=$ $B \times_{b_{1}} F_{1} \times{ }_{b_{2}} F_{2} \cdots \times_{b_{m}} F_{m}$ be a multiply twisted product and $P \in$ $\Gamma(T B)$; then the scalar curvature $\bar{S}$ has the following expression:

$$
\begin{aligned}
\bar{S}= & \bar{S}^{B}+2 \sum_{i=1}^{m} \frac{l_{i}}{b_{i}} \Delta_{B} b_{i}+\sum_{i=1}^{m} \frac{S^{F_{i}}}{b_{i}^{2}}+\sum_{i=1}^{m} l_{i}\left(l_{i}-1\right) \frac{\left|\operatorname{grad}_{B} b_{i}\right|_{B}^{2}}{b_{i}^{2}} \\
& +\sum_{i=1}^{m} \sum_{j \neq i} l_{i} l_{j} \frac{g_{B}\left(\operatorname{grad}_{B} b_{i}, \operatorname{grad}_{B} b_{j}\right)}{b_{i} b_{j}} \\
& +\sum_{i=1}^{m} l_{i}\left(n+\bar{n}+l_{i}-2\right) \frac{P\left(b_{i}\right)}{b_{i}} \\
& +\sum_{i=1}^{m} \sum_{j \neq i} l_{i} l_{j} \frac{P\left(b_{j}\right)}{b_{j}}+\sum_{i=1}^{m} l_{i}(n+\bar{n}-3) \pi(P) \\
& +2 \sum_{i=1}^{m} l_{i} \operatorname{div}_{B} P .
\end{aligned}
$$

By Proposition 9 and the definition of the scalar curvature, we have the following. 
Proposition 13 (compare with [5, Proposition 2.6]). Let $M=$ $B \times{ }_{b_{1}} F_{1} \times_{b_{2}} F_{2} \cdots \times_{b_{m}} F_{m}$ be a multiply twisted product and $P \in$ $\Gamma\left(T F_{r}\right)$; then the scalar curvature $\bar{S}$ has the following expression:

$$
\begin{aligned}
\bar{S}= & S^{B}+2 \sum_{i=1}^{m} \frac{l_{i}}{b_{i}} \Delta_{B} b_{i}+\sum_{i=1}^{m} \frac{S^{F_{i}}}{b_{i}^{2}}+\sum_{i=1}^{m} l_{i}\left(l_{i}-1\right) \frac{\left|\operatorname{grad}_{B} b_{i}\right|_{B}^{2}}{b_{i}^{2}} \\
& +\sum_{i=1}^{m} \sum_{j \neq i} l_{i} l_{j} \frac{g_{B}\left(\operatorname{grad}_{B} b_{i}, \operatorname{grad}_{B} b_{j}\right)}{b_{i} b_{j}}+\pi(P)(\bar{n}-1)(\bar{n}-2) \\
& +2(\bar{n}-1) \operatorname{div}_{F_{r}} P .
\end{aligned}
$$

Remark 14. When for each $i \in\{1, \ldots, m\}, b_{i}: B \rightarrow(0, \infty)$ is smooth and $P=0$, we get Proposition 2.6 in [5] by Propositions 12 and 13.

\section{Special Multiply Warped Product with a Semisymmetric Connection}

Let $M=I \times_{b_{1}} F_{1} \times_{b_{2}} F_{2} \cdots \times_{b_{m}} F_{m}$ be a multiply warped product with the metric tensor $-d t^{2} \oplus b_{1}^{2} g_{F_{1}} \oplus \cdots \oplus b_{m}^{2} g_{F_{m}}$ and $I$ is an open interval in $\mathbb{R}$ and $b_{i} \in C^{\infty}(I)$.

Theorem 15. Let $M=I \times_{b_{1}} F_{1} \times_{b_{2}} F_{2} \cdots \times_{b_{m}} F_{m}$ be a multiply warped product with the metric tensor $-d t^{2} \oplus b_{1}^{2} g_{F_{1}} \oplus \cdots \oplus b_{m}^{2} g_{F_{m}}$ and $P=(\partial / \partial t)$. Then $(M, \bar{\nabla})$ is Einstein with the Einstein constant $\lambda$ if and only if the following conditions are satisfied for any $i \in\{1, \ldots, m\}$ :

(1) $\left(F_{i}, \nabla^{F_{i}}\right)$ is Einstein with the Einstein constant $\lambda_{i}, i \in$ $\{1, \ldots, m\}$

(2) $\sum_{i=1}^{m} l_{i}\left(b_{i}^{\prime} / b_{i}-b_{i}^{\prime \prime} / b_{i}\right)=\lambda$;

(3) $\lambda_{i}-b_{i} b_{i}^{\prime \prime}-\left(l_{i}-1\right) b_{i}^{\prime 2}+\left(b_{i}^{2}-b_{i} b_{i}^{\prime}\right) \sum_{j \neq i} l_{j}\left(b_{j}^{\prime} / b_{j}\right)+(2-$ $\bar{n}) b_{i}^{2}+\left(\bar{n}+l_{i}-2\right) b_{i} b_{i}^{\prime}=\lambda b_{i}^{2}$.

Proof. By Proposition 7, we have

$$
\begin{gathered}
\overline{\operatorname{Ric}}\left(\frac{\partial}{\partial t}, \frac{\partial}{\partial t}\right)=-\sum_{i=1}^{m} l_{i}\left(\frac{b_{i}^{\prime}}{b_{i}}-\frac{b_{i}^{\prime \prime}}{b_{i}}\right) ; \\
\overline{\operatorname{Ric}}\left(\frac{\partial}{\partial t}, V\right)=\overline{\operatorname{Ric}}\left(V, \frac{\partial}{\partial t}\right)=0 ; \\
\overline{\operatorname{Ric}}(V, W)=\operatorname{Ric}^{F_{i}}(V, W)+g_{F_{i}}(V, W) \\
\times\left[-b_{i} b_{i}^{\prime \prime}-\left(l_{i}-1\right) b_{i}^{\prime 2}+\left(b_{i}^{2}-b_{i} b_{i}^{\prime}\right) \sum_{j \neq i} l_{j} \frac{b_{j}^{\prime}}{b_{j}}\right. \\
\left.+(2-\bar{n}) b_{i}^{2}+\left(\bar{n}+l_{i}-2\right) b_{i} b_{i}^{\prime}\right] .
\end{gathered}
$$

By (8) and the Einstein condition, we get Theorem 15.
Theorem 16. Let $M=I \times_{b_{1}} F_{1} \times_{b_{2}} F_{2} \cdots \times_{b_{m}} F_{m}$ be a multiply warped product with the metric tensor $-d t^{2} \oplus b_{1}^{2} g_{F_{1}} \oplus \cdots \oplus b_{m}^{2} g_{F_{m}}$ and $P \in \Gamma\left(T_{r}\right)$ with $g_{F_{r}}(P, P)=1$ and $\bar{n}>2$. Then $(M, \bar{\nabla})$ is Einstein with the Einstein constant $\lambda$ if and only if the following conditions are satisfied for any $i \in\{1, \ldots, m\}$ :

(1) $\left(F_{i}, \nabla^{F_{i}}\right)(i \neq r)$ is Einstein with the Einstein constant $\lambda_{i}$, $i \in\{1, \ldots, m\}$;

(2) $b_{r}$ is a constant and $\sum_{i=1}^{m} l_{i}\left(b_{i}^{\prime \prime} / b_{i}\right)=\mu_{0} ; \operatorname{div}_{F_{r}} P=$ $\mu_{1}, \mu_{0}-\mu_{1}+\lambda=(2-\bar{n}) b_{r}^{2}$, where $\mu_{0}, \mu_{1}$ are constants;

(3) $\operatorname{Ric}^{F_{r}}(V, W)+\bar{\lambda} g_{F_{r}}(V, W)=(\bar{n}-2)[\pi(V) \pi(W)-$ $\left.g\left(W, \nabla_{V} P\right)\right]$, for $V, W \in \Gamma\left(T F_{r}\right)$;

(4) $\lambda_{i}-b_{i} b_{i}^{\prime \prime}+(\bar{n}-2) b_{i}^{2} b_{r}^{2}-b_{i} b_{i}^{\prime} \sum_{j \neq i} l_{j}\left(b_{j}^{\prime} / b_{j}\right)-\left(l_{i}-1\right) b_{i}^{\prime 2}=$ $\left(\lambda-\mu_{1}\right) b_{i}^{2}$.

Proof. By Proposition 9(2) and $g_{F_{r}}(P, P)=1$, we have that $b_{r}$ is a constant. By Proposition 9, then

$$
\overline{\operatorname{Ric}}\left(\frac{\partial}{\partial t}, \frac{\partial}{\partial t}\right)=\sum_{i=1}^{m} l_{i} \frac{b_{i}^{\prime \prime}}{b_{i}}+(2-\bar{n}) b_{r}^{2}-\operatorname{div}_{F_{r}} P=-\lambda .
$$

By variables separation, we have

$$
\begin{aligned}
& \sum_{i=1}^{m} l_{i} \frac{b_{i}^{\prime \prime}}{b_{i}}=\mu_{0}, \\
& \operatorname{div}_{F_{r}} P=\mu_{1}, \quad \mu_{0}-\mu_{1}+\lambda=(2-\bar{n}) b_{r}^{2}, \\
& \overline{\operatorname{Ric}}(V, W)=\operatorname{Ric}^{F_{i}}(V, W)+b_{i}^{2} g_{F_{i}}(V, W) \\
& \times\left[-\frac{b_{i}^{\prime \prime}}{b_{i}}+\left(l_{i}-1\right) \frac{-b_{i}^{\prime 2}}{b_{i}^{2}}+\sum_{j \neq i} l_{j} \frac{-b_{i}^{\prime} b_{j}^{\prime}}{b_{i} b_{j}}\right. \\
& +(\bar{n}-2) \pi(P)]+(\bar{n}-2) g\left(W, \nabla_{V} P\right) \\
& +(2-\bar{n}) \pi(V) \pi(W)+g(V, W) \operatorname{div}_{F_{r}} P .
\end{aligned}
$$

When $i \neq r$, then $\nabla_{V} P=\pi(V)=0$, so

$$
\begin{aligned}
& \overline{\operatorname{Ric}}(V, W)= \operatorname{Ric}^{F_{i}}(V, W)+b_{i}^{2} g_{F_{i}}(V, W) \\
& \times\left[-\frac{b_{i}^{\prime \prime}}{b_{i}}+\left(l_{i}-1\right) \frac{-b_{i}^{\prime 2}}{b_{i}^{2}}+\sum_{j \neq i} l_{j} \frac{-b_{i}^{\prime} b_{j}^{\prime}}{b_{i} b_{j}}\right. \\
&\left.+(\bar{n}-2) b_{r}^{2}\right]+\mu_{1} b_{i}^{2} g_{F_{i}}(V, W)
\end{aligned}
$$

$$
=\lambda b_{i}^{2} g_{F_{i}}(V, W) \text {. }
$$


By variables separation, we have that $\left(F_{i}, \nabla^{F_{i}}\right)(i \neq r)$ is Einstein with the Einstein constant $\lambda_{i}$ and

$$
\begin{aligned}
\lambda_{i} & -b_{i} b_{i}^{\prime \prime}+(\bar{n}-2) b_{i}^{2} b_{r}^{2}-b_{i} b_{i}^{\prime} \sum_{j \neq i} l_{j} \frac{b_{j}^{\prime}}{b_{j}}-\left(l_{i}-1\right) b_{i}^{\prime 2} \\
& =\left(\lambda-\mu_{1}\right) b_{i}^{2} .
\end{aligned}
$$

When $i=r$ and $b_{r}$ is a constant, then

$$
\begin{gathered}
\operatorname{Ric}^{F_{i}}(V, W)+b_{r}^{2}\left[(\bar{n}-2) b_{r}^{2}+\mu_{1}-\lambda\right] g_{F_{i}}(V, W) \\
=(\bar{n}-2)\left[\pi(V) \pi(W)-g\left(W, \nabla_{V} P\right)\right] .
\end{gathered}
$$

So we prove the above theorem.

Remark 17. Comparing with Theorem 3.3 in [5], in Theorems 15 and 16, the unit vector field $P$ emerges. For Theorem 3.3 in [5], $P$ equals zero. So Theorem 3.3 in [5] is not the special case of Theorems 15 and 16. Then equations in our theorems are different from the equations in theorems in [5].

When $M=I \times_{b_{1}} F_{1} \times_{b_{2}} F_{2} \cdots \times_{b_{m}} F_{m}$ is a multiply warped product and $P=\partial / \partial t$, by Proposition 12, we have

$$
\begin{aligned}
\bar{S}= & -2 \sum_{i=1}^{m} l_{i} \frac{b_{i}^{\prime \prime}}{b_{i}}+\sum_{i=1}^{m} \frac{S^{F_{i}}}{b_{i}^{2}}+\sum_{i=1}^{m} l_{i}\left(l_{i}-1\right) \frac{-b_{i}^{\prime 2}}{b_{i}^{2}} \\
& +\sum_{i=1}^{m} \sum_{j \neq i} l_{i} l_{j} \frac{-b_{i} b_{j}}{b_{i} b_{j}}+\sum_{i=1}^{m} l_{i}\left(\bar{n}+l_{i}-1\right) \frac{b_{i}^{\prime}}{b_{i}} \\
& +\sum_{i=1}^{m} \sum_{j \neq i} l_{i} l_{j} \frac{b_{j}^{\prime}}{b_{j}}-\sum_{i=1}^{m} l_{i}(\bar{n}-2) .
\end{aligned}
$$

The following result just follows from the method of separation of variables and the fact that each $S^{F_{i}}$ is function defined on $F_{i}$.

Proposition 18. Let $M=I \times_{b_{1}} F_{1} \times_{b_{2}} F_{2} \cdots \times_{b_{m}} F_{m}$ be a multiply warped product and $P=\partial / \partial t$. If $(M, \bar{\nabla})$ has constant scalar curvature $\bar{S}$, then each $\left(F_{i}, \nabla^{F_{i}}\right)$ has constant scalar curvature $S^{F_{i}}$.

When $P \in \Gamma\left(T F_{r}\right)$, by Proposition 13, we have

$$
\begin{aligned}
\bar{S}= & -2 \sum_{i=1}^{m} l_{i} \frac{b_{i}^{\prime \prime}}{b_{i}}+\sum_{i=1}^{m} \frac{S^{F_{i}}}{b_{i}^{2}}+\sum_{i=1}^{m} l_{i}\left(l_{i}-1\right) \frac{-b_{i}^{\prime 2}}{b_{i}^{2}} \\
& +\sum_{i=1}^{m} \sum_{j \neq i} l_{i} l_{j} \frac{-b_{i}^{\prime} b_{j}^{\prime}}{b_{i} b_{j}}+\pi(P)(\bar{n}-1)(\bar{n}-2) \\
& +2(\bar{n}-1) \operatorname{div}_{F_{r}} P .
\end{aligned}
$$

Similarly we have the following.

Proposition 19 (compare with [5, Proposition 3.5]). Let $M=$ $I \times_{b_{1}} F_{1} \times_{b_{2}} F_{2} \cdots \times_{b_{m}} F_{m}$ be a multiply warped product and $P \in$ $\Gamma\left(T F_{r}\right)$. If $(M, \bar{\nabla})$ has constant scalar curvature $\bar{S}$, then
(1) each $\left(F_{i}, \nabla^{F_{i}}\right)(i \neq r)$ has constant scalar curvature $S^{F_{i}}$;

(2) moreover, if $g_{F_{r}}(P, P), \operatorname{div}_{F_{r}} P$ are also constants, then $S^{F_{r}}$ is a constant.

Remark 20. When $P=0$ in Proposition 19, we get Proposition 3.5 in [5].

\section{Generalized Robertson-Walker Space-Times with a Semisymmetric Metric Connection}

In this section, we study $M=I \times F$ with the metric tensor $-d t^{2}+f(t)^{2} g_{F}$. As a corollary of Theorem 15, we obtain the following.

Corollary 21. Let $M=I \times F$ with the metric tensor $-d t^{2}+$ $f(t)^{2} g_{F}$ and $P=\partial / \partial t$. Then $(M, \bar{\nabla})$ is Einstein with the Einstein constant $\lambda$ if and only if the following conditions are satisfied:

(1) $\left(F, \nabla^{F}\right)$ is Einstein with the Einstein constant $\lambda_{F}$;

(2) $l\left(\left(f^{\prime} / f\right)-\left(f^{\prime \prime} / f\right)\right)=\lambda$;

(3) $\lambda_{F}-f f^{\prime \prime}+(1-l) f^{\prime 2}+(1-l-\lambda) f^{2}+(2 l-1) f^{\prime} f=0$.

Remark 22. In Theorem 5.1 in [9], they got the Einstein condition of $M=I \times F$ with a semisymmetric metric connection, but they did not consider conditions (2) and (3).

Corollary 23. Let $M=I \times F$ with the metric tensor $-d t^{2}+$ $f(t)^{2} g_{F}$ and $P=\partial / \partial t$ and $\operatorname{dim} F=1$. Then $(M, \bar{\nabla})$ is Einstein with the Einstein constant $\lambda$ if and only if $f^{\prime \prime}=f^{\prime}-\lambda f$.

By Corollary 21 (2) and (3), we get the following.

Corollary 24. Let $M=I \times F$ with the metric tensor $-d t^{2}+$ $f(t)^{2} g_{F}$ and $P=\partial / \partial t$ and $\operatorname{dim} F>1$. Then $(M, \bar{\nabla})$ is Einstein with the Einstein constant $\lambda$ if and only if the following conditions are satisfied:

(1) $\left(F, \nabla^{F}\right)$ is Einstein with the Einstein constant $\lambda_{F}$;

(2) $f^{\prime \prime}=f^{\prime}-(\lambda / l) f$;

(3) $\left(\lambda_{F} /(1-l)\right)+f^{\prime 2}+(1+(\lambda / l)) f^{2}-2 f f^{\prime}=0$.

By Corollary 23 and elementary methods for ordinary differential equations, we get the following.

Theorem 25. Let $M=I \times F$ with the metric tensor $-d t^{2}+$ $f(t)^{2} g_{F}$ and $P=\partial / \partial t$ and $\operatorname{dim} F=1$. Then $(M, \bar{\nabla})$ is Einstein with the Einstein constant $\lambda$ if and only if

(1) $\lambda<1 / 4, f(t)=c_{1} e^{((1+\sqrt{1-4 \lambda}) / 2) t}+c_{2} e^{((1-\sqrt{1-4 \lambda}) / 2) t}$,

(2) $\lambda=1 / 4, f(t)=c_{1} e^{(1 / 2) t}+c_{2} t e^{(1 / 2) t}$,

(3) $\lambda>1 / 4, f(t)=c_{1} e^{(1 / 2) t} \cos ((\sqrt{4 \lambda-1} / 2) t)+$ $c_{2} e^{(1 / 2) t} \sin ((\sqrt{4 \lambda-1} / 2) t)$. 
Let $\lambda / l=d_{0}, \lambda_{F} /(1-l)=\overline{d_{0}},\left(1+\sqrt{1-4 d_{0}}\right) / 2=a_{0}, \quad(1-$ $\left.\sqrt{1-4 d_{0}}\right) / 2=b_{0}$; then, $a_{0}+b_{0}=1, d_{0}=a_{0} b_{0}$. When $\operatorname{dim} F>$ 1 , by Corollary 24(2), we have the following three cases.

Case (i) $\left(d_{0}<1 / 4\right)$. We have $f=c_{1} e^{a_{0} t}+c_{2} e^{b_{0} t}$. By Corollary 24(3), then

$$
\begin{aligned}
\overline{d_{0}} & +c_{1}^{2}\left(a_{0}^{2}+1+a_{0} b_{0}-2 a_{0}\right) e^{2 a_{0} t} \\
& +c_{2}^{2}\left(b_{0}^{2}+1+a_{0} b_{0}-2 b_{0}\right) e^{2 b_{0} t} \\
& +2 c_{1} c_{2}\left(2 a_{0} b_{0}+1-a_{0}-b_{0}\right) e^{\left(a_{0}+b_{0}\right) t}=0 .
\end{aligned}
$$

When $b_{0}=0$, we get $d_{0}=0, a_{0}=1, \lambda=0$. By (16), $\overline{d_{0}}+$ $c_{2}^{2}=0$, so $\lambda_{F}=(l-1) c_{2}^{2}$. In this case $f=c_{1} e^{t}+c_{2}$. When $b \neq 0$, then $e^{2 a_{0} t}, e^{2 b_{0} t}$, and $e^{\left(a_{0}+b_{0}\right) t}$ are linearly independent, so $c_{2}^{2}\left(b_{0}^{2}+1+a_{0} b_{0}-2 b_{0}\right)=c_{2}^{2}\left(1-b_{0}\right)=0$ and $c_{2}=0$. Then $c_{1} \neq 0$, by $c_{1}^{2}\left(a_{0}^{2}+1+a_{0} b_{0}-2 a_{0}\right)=c_{1}^{2}\left(1-a_{0}\right)=0$, so $a_{0}=1$; then $d_{0}=\lambda=0$. Thus $f=c_{1} e^{t}$.

Case (ii) $\left(d_{0}=1 / 4\right)$. One has $f=c_{1} e^{(1 / 2) t}+c_{2} t e^{(1 / 2) t}$. By Corollary 24(3), then

$$
\begin{aligned}
\overline{d_{0}}+e^{t}\left[\left(\frac{1}{2} c_{1}+c_{2}+\frac{1}{2} c_{2} t\right)^{2}+\frac{5}{4}\left(c_{1}+c_{2} t\right)^{2}\right. \\
\left.-2\left(\frac{1}{2} c_{1}+c_{2}+\frac{1}{2} c_{2} t\right)\left(c_{1}+c_{2} t\right)\right]=0 .
\end{aligned}
$$

The coefficient of $t^{2} e^{t}$ is $(1 / 2) c_{2}^{2}$, so $c_{2}=0$. The coefficient of $e^{t}$ is $\left((1 / 2) c_{1}+c_{2}\right)^{2}+(5 / 4)\left(c_{1}\right)^{2}-2 c_{1}\left((1 / 2) c_{1}+c_{2}\right)$, so $c_{1}=0$; in this case we have no solutions.

Case (iii) $\left(d_{0}>1 / 4\right)$. One has $f(t)=c_{1} e^{(1 / 2) t} \cos \left(h_{0} t\right)+$ $c_{2} e^{(1 / 2) t} \sin \left(h_{0} t\right)$, where $h_{0}=\sqrt{4 d_{0}-1} / 2$. By Corollary 24(3), then

$$
\begin{aligned}
\overline{d_{0}}+e^{t}\{ & {\left[\left(\frac{c_{1}}{2}+c_{2} h_{0}\right) \cos \left(h_{0} t\right)+\left(\frac{c_{2}}{2}-c_{1} h_{0}\right) \sin \left(h_{0} t\right)\right]^{2} } \\
& +\left(1+d_{0}\right)\left(c_{1} \cos \left(h_{0} t\right)+c_{2} \sin \left(h_{0} t\right)\right)^{2} \\
& -2\left(c_{1} \cos \left(h_{0} t\right)+c_{2} \sin \left(h_{0} t\right)\right) \\
& \left.\times\left[\left(\frac{c_{1}}{2}+c_{2} h_{0}\right) \cos \left(h_{0} t\right)+\left(\frac{c_{2}}{2}-c_{1} h_{0}\right) \sin \left(h_{0} t\right)\right]\right\}
\end{aligned}
$$

$=0$.

Considering the coefficients of $\cos ^{2}\left(h_{0} t\right) e^{t}$ and $\sin ^{2}\left(h_{0} t\right) e^{t}$, we get

$$
\begin{aligned}
& \left(\frac{1}{4}+d_{0}\right) c_{1}^{2}+c_{2}^{2} h_{0}^{2}-c_{1} c_{2} h_{0}=0 \\
& \left(\frac{1}{4}+d_{0}\right) c_{2}^{2}+c_{1}^{2} h_{0}^{2}+c_{1} c_{2} h_{0}=0
\end{aligned}
$$

Adding the above two equalities, then $1 / 4+d_{0}+h_{0}^{2}=0$ and $d_{0}=0$. There is a contradiction with $d_{0}>1 / 4$ and in this case we have no solutions. So we obtain the following theorem.
Theorem 26. Let $M=I \times F$ with the metric tensor $-d t^{2}+$ $f(t)^{2} g_{F}$ and $P=\partial / \partial t$ and $\operatorname{dim} F>1$. Then $(M, \bar{\nabla})$ is Einstein with the Einstein constant $\lambda$ if and only if $\lambda=0$ and $f=c_{1} e^{t}+c_{2}$ and $\left(F, \nabla^{F}\right)$ is Einstein with the Einstein constant $(l-1) c_{2}^{2}$.

By (14) and (15), we have the following.

Corollary 27. Let $M=I \times F$ with the metric tensor $-d t^{2}+$ $f(t)^{2} g_{F}$ and $P=\partial / \partial t$. Then $(M, \bar{\nabla})$ has constant scalar curvature $\bar{S}$ if and only if $\left(F, \nabla^{F}\right)$ has constant scalar curvature $S^{F}$ and

$$
\bar{S}=\frac{S^{F}}{f^{2}}-2 l \frac{f^{\prime \prime}}{f}-l(l-1) \frac{f^{\prime 2}}{f^{2}}+2 l^{2} \frac{f^{\prime}}{f}+(1-l) l .
$$

Corollary 28. Let $M=I \times F$ with the metric tensor $-d t^{2}+$ $f(t)^{2} g_{F}$ and $P \in \Gamma(T F)$ and $g_{F}(P, P)=c_{0}$, $\operatorname{div}_{F_{r}} P=c_{0}^{\prime}$, where $c_{0}$ and $c_{0}^{\prime}$ are constants. Then $(M, \bar{\nabla})$ has constant scalar curvature $\bar{S}$ if and only if $\left(F, \nabla^{F}\right)$ has constant scalar curvature $S^{F}$ and

$$
\bar{S}=\frac{S^{F}}{f^{2}}-2 l \frac{f^{\prime \prime}}{f}-l(l-1) \frac{f^{\prime 2}}{f^{2}}+c_{0}(l-1) l f^{2}+2 c_{0}^{\prime} l .
$$

In (20), we make the change of variable $f(t)=\sqrt{v(t)}$ and have the following equation:

$$
v^{\prime \prime}(t)+\frac{l-3}{4} \frac{v^{\prime}(t)^{2}}{v(t)}-l v^{\prime}(t)+\left(l-1+\frac{\bar{S}}{l}\right) v(t)-\frac{S^{F}}{l}=0 .
$$

Theorem 29. Let $M=I \times F$ with the metric tensor $-d t^{2}+$ $f(t)^{2} g_{F}$ and $P=\partial / \partial t$ and $\operatorname{dim} F=l=3$. Then $(M, \bar{\nabla})$ has constant scalar curvature $\bar{S}$ if and only if $\left(F, \nabla^{F}\right)$ has constant scalar curvature $S^{F}$

$$
\begin{aligned}
& \text { (1) } \bar{S}<3 / 4 \text { and } \bar{S} \neq-6, v(t)=c_{1} e^{((3+\sqrt{1-(4 / 3) \bar{S}}) / 2) t}+ \\
& c_{2} e^{((3-\sqrt{1-(4 / 3) \bar{S}}) / 2) t}+\left(S^{F} /(6+\bar{S})\right) ; \\
& \text { (2) } \bar{S}=3 / 4, \quad v(t)=c_{1} e^{(3 / 2) t}+c_{2} t e^{(3 / 2) t}+\left(S^{F} /(6+\bar{S})\right) ; \\
& \text { (3) } \bar{S}>3 / 4, \quad v(t)=c_{1} e^{(3 / 2) t} \cos ((\sqrt{(4 / 3) \bar{S}-1 / 2}) t)+ \\
& c_{2} e^{(3 / 2) t} \sin \left((\sqrt{(4 / 3) \bar{S}-1 / 2) t})+\left(S^{F} /(6+\bar{S})\right) ;\right.
\end{aligned}
$$

(4) $\bar{S}=-6, v(t)=c_{1}-\left(S^{F} / 9\right) t+c_{2} e^{3 t}$.

Proof. If $l=3$, then we have a simple differential equation as follows:

$$
v^{\prime \prime}(t)-3 v^{\prime}(t)+\left(2+\frac{\bar{S}}{3}\right) v(t)-\frac{S^{F}}{3}=0
$$

If $\bar{S} \neq-6$, putting $h(t)=(2+\bar{S} / 3) v(t)-S^{F} / 3$, it follows that $h^{\prime \prime}(t)-3 h^{\prime}(t)+(2+\bar{S} / 3) h(t)=0$. The above solutions (1)-(3) follow directly from elementary methods for ordinary differential equations. When $\bar{S}=-6$, then $v^{\prime \prime}(t)-3 v^{\prime}(t)-$ $S^{F} / 3=0$, and we get solution (4). 
Theorem 30. Let $M=I \times F$ with the metric tensor $-d t^{2}+$ $f(t)^{2} g_{F}$ and $P=\partial / \partial t$ and $\operatorname{dim} F=l \neq 3$ and $S^{F}=0 . \operatorname{If}(M, \bar{\nabla})$ has constant scalar curvature $\bar{S}$ if and only if

(1) $\bar{S}<l /(l+1), v(t)=\left(c_{1} e^{((l+\sqrt{1-((l+1) / l) \bar{S}}) / 2) t}+\right.$ $\left.c_{2} e^{((l-\sqrt{1-((l+1) / l) \bar{S}}) / 2) t}\right)^{4 /(l+1)}$;

(2) $\bar{S}=l /(l+1), v(t)=\left(c_{1} e^{(l / 2) t}+c_{2} t e^{(l / 2) t}\right)^{4 /(l+1)}$;

(3) $\bar{S} l /(l+1), v(t)$

$\left(c_{1} e^{(l / 2) t} \cos ((\sqrt{((l+1) / l) \bar{S}-1} / 2) t)\right.$

$=$

$\left.c_{2} e^{(l / 2) t} \sin ((\sqrt{((l+1) / l) \bar{S}-1} / 2) t)\right)^{4 /(l+1)}$.

Proof. In this case, (22) is changed into the simpler form

$$
\frac{v^{\prime \prime}(t)}{v(t)}+\frac{l-3}{4} \frac{v^{\prime}(t)^{2}}{v(t)^{2}}-l \frac{v^{\prime}(t)}{v(t)}+\left(l-1+\frac{\bar{S}}{l}\right)=0 .
$$

Putting $v(t)=w(t)^{4 /(l+1)}$, then $w(t)$ satisfies the equation $w^{\prime \prime}-$ $l w^{\prime}+((l+1) / 4)(l-1+\bar{S} / l) w=0$; by the elementary methods for ordinary differential equations we prove the above theorem.

When $\operatorname{dim} F=l \neq 3$ and $S^{F} \neq 0$, putting $v(t)=w(t)^{4 /(l+1)}$, then $w(t)$ satisfies the following equation:

$$
w^{\prime \prime}-l w^{\prime}+\frac{(l+1)}{4}\left(l-1+\frac{\bar{S}}{l}\right) w-\frac{(l+1)}{4} \frac{S^{F}}{l} w^{1-4 /(l+1)}=0 .
$$

\section{Generalized Kasner Space-Times with a Semisymmetric Metric Connection}

In this section, we consider the scalar and Ricci curvature of generalized Kasner space-times with a semisymmetric metric connection. We recall the definition of generalized Kasner space-times [5].

Definition 31. A generalized Kasner space-time $(M, g)$ is a Lorentzian multiply warped product of the form $M=$ $I \times_{\phi^{p_{1}}} F_{1} \times \cdots \times_{\phi^{p_{m}}} F_{m}$ with the metric $g=-d t^{2} \oplus \phi^{2 p_{1}} g_{F_{1}} \oplus$ $\cdots \oplus \phi^{2 p_{m}} g_{F_{m}}$, where $\phi: I \rightarrow(0, \infty)$ is smooth and $p_{i} \in \mathbb{R}$, for any $i \in\{1, \ldots, m\}$ and also $I=\left(t_{1}, t_{2}\right)$.

We introduce the following parameters $\zeta=\sum_{i=1}^{m} l_{i} p_{i}$ and $\eta=\sum_{i=1}^{m} l_{i} p_{i}^{2}$ for generalized Kasner space-times. By Theorem 15 and direct computations, we get the following.

Proposition 32. Let $M=I \times_{\phi^{p_{1}}} F_{1} \times \cdots \times_{\phi^{p_{m}}} F_{m}$ be a generalized Kasner space-time and $P=\partial / \partial t$. Then $(M, \bar{\nabla})$ is Einstein with the Einstein constant $\lambda$ if and only if the following conditions are satisfied for any $i \in\{1, \ldots, m\}$ :

(1) $\left(F_{i}, \nabla^{F_{i}}\right)$ is Einstein with the Einstein constant $\lambda_{i}, i \in$ $\{1, \ldots, m\}$;

(2) $\zeta\left(\left(\phi^{\prime}-\phi^{\prime \prime}\right) / \phi\right)-(\eta-\zeta)\left(\phi^{\prime 2} / \phi^{2}\right)=\lambda$;

(3) $\lambda_{i} / \phi^{2 p_{i}}-p_{i}\left(\phi^{\prime \prime} / \phi\right)-(\zeta-1) p_{i}\left(\phi^{\prime 2} / \phi^{2}\right)+[\zeta+(\bar{n}-$ 2) $\left.p_{i}\right]\left(\phi^{\prime} / \phi\right)=\bar{n}+\lambda-2$.
By (14), we obtain the following.

Proposition 33. Let $M=I \times_{\phi^{p_{1}}} F_{1} \times \cdots \times_{\phi^{p_{m}}} F_{m}$ be $a$ generalized Kasner space-time and $P=\partial / \partial t$. Then $(M, \bar{\nabla})$ has constant scalar curvature $\bar{S}$ if and only if each $\left(F_{i}, \nabla^{F_{i}}\right)$ has constant scalar curvature $S^{F_{i}}$ and

$$
\begin{aligned}
\bar{S}= & \sum_{i=1}^{m} \frac{S^{F_{i}}}{\phi^{2 p_{i}}}-2 \zeta \frac{\phi^{\prime \prime}}{\phi}-\left(\eta+\zeta^{2}-2 \zeta\right) \frac{\phi^{\prime 2}}{\phi^{2}}+2(\bar{n}-1) \zeta \frac{\phi^{\prime}}{\phi} \\
& +(2-\bar{n})(\bar{n}-1) .
\end{aligned}
$$

Next, we first give a classification of four-dimensional generalized Kasner space-times with a semisymmetric metric connection and then consider Ricci tensors and scalar curvatures of them.

Definition 34. Let $M=I \times_{b_{1}} F_{1} \times \cdots \times_{b_{m}} F_{m}$ with the metric $g=-d t^{2} \oplus b_{1}^{2} g_{F_{1}} \oplus \cdots \oplus b_{m}^{2} g_{F_{m}}$. Consider the following:

(i) $(M, g)$ is said to be of type (I) if $m=1$ and $\operatorname{dim}(F)=3$;

(ii) $(M, g)$ is said to be of type (II) if $m=2$ and $\operatorname{dim}\left(F_{1}\right)=$ 1 and $\operatorname{dim}\left(F_{2}\right)=2$;

(iii) $(M, g)$ is said to be of type (III) if $m=3$ and $\operatorname{dim}\left(F_{1}\right)=$ $1, \operatorname{dim}\left(F_{2}\right)=1$ and $\operatorname{dim}\left(F_{3}\right)=1$.

By Theorems 26 and 29, we have given a classification of type (I) Einstein spaces and type (I) spaces with the constant scalar curvature.

5.1. Classification of Einstein Type (II) Generalized Kasner Space-Times with a Semisymmetric Metric Connection. Let $M=I \times_{\phi^{p_{1}}} F_{1} \times_{\phi^{p_{2}}} F_{2}$ be an Einstein type (II) generalized Kasner space-time and $P=\partial / \partial t$. Then $\zeta=p_{1}+2 p_{2}, \eta=$ $p_{1}^{2}+2 p_{2}^{2}$. By Theorem 15, we have

$$
\begin{gathered}
\zeta\left(\frac{\phi^{\prime}-\phi^{\prime \prime}}{\phi}\right)-(\eta-\zeta) \frac{\phi^{\prime 2}}{\phi^{2}}=\lambda, \\
-p_{1} \frac{\phi^{\prime \prime}}{\phi}-(\zeta-1) p_{1} \frac{\phi^{\prime 2}}{\phi^{2}}+\left[\zeta+2 p_{1}\right] \frac{\phi^{\prime}}{\phi}=\lambda+2, \\
\frac{\lambda_{2}}{\phi^{2} p_{2}}-p_{2} \frac{\phi^{\prime \prime}}{\phi}-(\zeta-1) p_{2} \frac{\phi^{\prime 2}}{\phi^{2}}+\left[\zeta+2 p_{2}\right] \frac{\phi^{\prime}}{\phi}=\lambda+2,
\end{gathered}
$$

where $\lambda_{2}$ is a constant. Consider the following two cases. Case (i) $(\zeta=0)$. In this case, $p_{2}=-(1 / 2) p_{1}, \eta=(3 / 2) p_{1}^{2}$. Then by (27a)-(5.2iii), we have

$$
\begin{gathered}
-\eta \frac{\phi^{\prime 2}}{\phi^{2}}=\lambda, \\
p_{1}\left(-\frac{\phi^{\prime \prime}}{\phi}+\frac{\phi^{\prime 2}}{\phi^{2}}+2 \frac{\phi^{\prime}}{\phi}\right)=\lambda+2, \\
\frac{\lambda_{2}}{\phi^{-p_{1}}}-\frac{1}{2} p_{1}\left(-\frac{\phi^{\prime \prime}}{\phi}+\frac{\phi^{\prime 2}}{\phi^{2}}+2 \frac{\phi^{\prime}}{\phi}\right)=\lambda+2 .
\end{gathered}
$$


Case (i) (a) $(\eta=0)$. One has $p_{i}=0$; by (28a), $\lambda=0$. By (28b), $\lambda+2=0$; this is a contradiction.

Case $(i)(b)(\eta \neq 0)$. One has $p_{i} \neq 0$.

Case (i)(b)(1) $\left(\lambda_{2}=0\right)$. By (28b) and (28c), $\lambda=-2$ and

$$
-\frac{\phi^{\prime \prime}}{\phi}+\frac{\phi^{\prime 2}}{\phi^{2}}+2 \frac{\phi^{\prime}}{\phi}=0, \quad \frac{\phi^{\prime 2}}{\phi^{2}}=\frac{2}{\eta} ;
$$

then $\phi=c_{0} e^{ \pm(\sqrt{2 / \eta}) t}$ which does not satisfy the first equation in (29); this a contradiction.

Case $(i)(b)(2)\left(\lambda_{2} \neq 0\right)$. By (28b) and (28c), we have $\lambda_{2} / \phi^{-p_{1}}=$ $(3 / 2)(\lambda+2)$, so $\phi$ is a constant. By $(28 \mathrm{~b}), \lambda+2=0$, so $\lambda_{2}=0$; this is a contradiction. In a word, we have no solutions when $\zeta=0$.

Case (ii) $(\zeta \neq 0)$. One has $\eta \neq 0$. Putting $\phi=\psi^{\zeta / \eta}$, then $\psi^{\prime \prime}-$ $\psi^{\prime}+\left(\lambda \eta / \zeta^{2}\right) \psi=0$. Hence,

$$
\begin{aligned}
& \text { (1) } \lambda<\zeta^{2} / 4 \eta, \quad \psi=c_{1} e^{\left(\left(1+\sqrt{1-4 \lambda \eta / \zeta^{2}}\right) / 2\right) t}+ \\
& c_{2} e^{\left(\left(1-\sqrt{1-4 \lambda \eta / \zeta^{2}}\right) / 2\right) t} \\
& \text { (2) } \lambda=\zeta^{2} / 4 \eta, \psi=c_{1} e^{(1 / 2) t}+c_{2} t e^{(1 / 2) t} \\
& \text { (3) } \lambda>\zeta^{2} / 4 \eta, \psi=c_{1} e^{(1 / 2) t} \cos \left(\left(\sqrt{4 \lambda \eta / \zeta^{2}-1} / 2\right) t\right)+ \\
& c_{2} e^{(1 / 2) t} \sin \left(\left(\sqrt{4 \lambda \eta / \zeta^{2}-1} / 2\right) t\right) .
\end{aligned}
$$

We make (27a), (5.2ii), (5.2iii) into

$$
\begin{gathered}
\frac{\zeta^{2}}{\eta} \frac{\psi^{\prime}-\psi^{\prime \prime}}{\psi}=\lambda, \quad \psi=\phi^{\eta / \zeta}, \\
-\frac{p_{1}}{\zeta} \frac{\left(\phi^{\zeta}\right)^{\prime \prime}}{\phi^{\zeta}}+\frac{\zeta+2 p_{1}}{\zeta} \frac{\left(\phi^{\zeta}\right)^{\prime}}{\phi^{\zeta}}=\lambda+2, \\
\frac{\lambda_{2}}{\phi^{2 p_{2}}}-\frac{p_{2}}{\zeta} \frac{\left(\phi^{\zeta}\right)^{\prime \prime}}{\phi^{\zeta}}+\frac{\zeta+2 p_{2}}{\zeta} \frac{\left(\phi^{\zeta}\right)^{\prime}}{\phi^{\zeta}}=\lambda+2 .
\end{gathered}
$$

When $p_{1}=p_{2}$, type (II) spaces turn into type (I) spaces, so we assume $p_{1} \neq p_{2}$. By (30b) and (30c), then

$$
\psi^{\prime}=\frac{p_{1} \lambda_{2} \eta}{\left(p_{2}-p_{1}\right) \zeta^{2}} \psi^{1-2 p_{2} \zeta / \eta}+\frac{(\lambda+2) \eta}{\zeta^{2}} \psi .
$$

Case (ii)(1).Consider $\lambda<\zeta^{2} / 4 \eta, \psi=c_{1} e^{a t}+c_{2} e^{b t}$, where $a=$ $\left(1+\sqrt{1-4 \lambda \eta / \zeta^{2}}\right) / 2, b=\left(\left(1-\sqrt{1-4 \lambda \eta / \zeta^{2}}\right) / 2\right) t$.

By (31),

$$
\begin{aligned}
a c_{1} e^{a t}+b c_{2} e^{b t} & \frac{p_{1} \lambda_{2} \eta}{\left(p_{2}-p_{1}\right) \zeta^{2}}\left(c_{1} e^{a t}+c_{2} e^{b t}\right)^{1-2 p_{2} \zeta / \eta} \\
& +\frac{(\lambda+2) \eta}{\zeta^{2}}\left(c_{1} e^{a t}+c_{2} e^{b t}\right)
\end{aligned}
$$

Case (ii)(1)(a) (c $\left.c_{1}=0\right)$. One has

$$
\left[b-\frac{(\lambda+2) \eta}{\zeta^{2}}\right] c_{2} e^{b t}=\frac{p_{1} \lambda_{2} \eta}{\left(p_{2}-p_{1}\right) \zeta^{2}}\left(c_{2} e^{b t}\right)^{1-2 p_{2} \zeta / \eta} .
$$

Case (ii)(1)(a)(1) $\left(b \neq 0, p_{1} \lambda_{2} \neq 0\right)$. One has $p_{2}=0$ and $\zeta=$ $p_{1}, \eta=p_{1}^{2}$ and $b=(1-\sqrt{1-4 \lambda}) / 2$ and $\psi=c_{2} e^{b t}$. By (30b) and $b^{2}-b+\lambda=0$, we get $-b^{2}+3 b=\lambda+2$ and $b=1$. But $b<1 / 2$, and this is a contradiction.

Case (ii)(1)(a)(2) $\left(b \neq 0, p_{1} \lambda_{2}=0\right)$. If $p_{1}=0$, then $\zeta=2 p_{2}$, $\eta=2 p_{2}^{2}$ and $b=(\lambda+2) \eta / \zeta^{2}$, so $\lambda=-4$ and $b=-1$. By (30c), we get $\lambda_{2}=0$ and $-2 b^{2}+4 b=\lambda+2$ which is a contradiction.

If $\lambda_{2}=0$, by (30b) and $b=(\lambda+2) \eta / \zeta^{2}$, we get $\lambda=0$ or -2 . When $\lambda=0$, then $b=2 \eta / \zeta^{2}=0$; this is a contradiction. There is a similar contradiction for $\lambda=-2$.

Case (ii)(1)(a)(3) $(b=0)$. One has $\psi=c_{2}$; by (30a), $\lambda=0$. By (30b), $\lambda=-2$; this is a contradiction.

Case (ii)(1)(b) $\left(c_{2}=0\right)$. One has

$$
\left[a-\frac{(\lambda+2) \eta}{\zeta^{2}}\right] c_{1} e^{a t}=\frac{p_{1} \lambda_{2} \eta}{\left(p_{2}-p_{1}\right) \zeta^{2}}\left(c_{1} e^{a t}\right)^{1-2 p_{2} \zeta / \eta} .
$$

Case $(i i)(1)(b)(1)\left(p_{1} \lambda_{2} \neq 0\right)$. One has $p_{2}=0$ and $\zeta=2 p_{2}, \eta=$ $2 p_{2}^{2}$ and $a=(1+\sqrt{1-4 \lambda}) / 2$ and $\lambda_{2}=\lambda+2-(1+\sqrt{1-4 \lambda}) / 2$. By (30b), then $a=1$ and $\lambda=0$, so $\lambda_{2}=1$ and $\psi=c_{1} e^{t}$ and $\phi$ satisfies (30c). In this case, we get $p_{2}=0, p_{1} \neq 0, \phi=$ $c_{0} e^{t / p_{1}}, \lambda=0, \lambda_{2}=1$.

Case (ii)(1)(b)(2) $\left(p_{1} \lambda_{2}=0\right)$. If $p_{1}=0$, then $\zeta=2 p_{2}, \eta=2 p_{2}^{2}$ and $\psi=c_{1} e^{a t}$ and $a=(\lambda+2) \eta / \zeta^{2}$, so $\lambda=0$ and $a=1$. By (30c), we get $\lambda_{2}=0$ and $\phi$ satisfies (30b) and (30c). In this case, $p_{1}=0, p_{2} \neq 0 \lambda=0, \lambda_{2}=0, \phi=c_{0} e^{t / p_{2}}$.

If $\lambda_{2}=0$, by (30b) and $a=(\lambda+2) \eta / \zeta^{2}$, then $\lambda=0$ and $a=2 \eta / \zeta^{2}=1$. By (30c), then $\lambda_{2}=0$ and $\phi$ satisfies (30b) and (30c). In this case, $p_{1} \neq 0, p_{2} \neq 0, \lambda=\lambda_{2}=0, p_{1}=4 p_{2}, \phi=$ $c_{0} e^{t / 3 p_{2}}$.

Case (ii)(1)(c) $\quad\left(c_{1} \neq 0, c_{2} \neq 0, b \neq 0\right)$. If $p_{2} \neq 0$, then $e^{a t}, e^{b t},\left(c_{1} e^{a t}+c_{2} e^{b t}\right)^{1-2 p_{2} \zeta / \eta}$ are linearly independent; by (32), then

$$
\begin{gathered}
{\left[a-\frac{(\lambda+2) \eta}{\zeta^{2}}\right] c_{1}=0, \quad\left[b-\frac{(\lambda+2) \eta}{\zeta^{2}}\right] c_{2}=0} \\
\frac{p_{1} \lambda_{2} \eta}{\left(p_{2}-p_{1}\right) \zeta^{2}}\left(c_{1} e^{a t}\right)^{1-2 p_{2} \zeta / \eta}
\end{gathered}
$$

So $a=b=(\lambda+2) \eta / \zeta^{2}$, and this is a contradiction.

If $p_{2}=0$, then by (32),

$$
\begin{aligned}
& a-\frac{(\lambda+2) \eta}{\zeta^{2}}-\frac{p_{1} \lambda_{2} \eta}{\left(p_{2}-p_{1}\right) \zeta^{2}}=0, \\
& b-\frac{(\lambda+2) \eta}{\zeta^{2}}-\frac{p_{1} \lambda_{2} \eta}{\left(p_{2}-p_{1}\right) \zeta^{2}}=0,
\end{aligned}
$$

so $a=b$ and we get a contradiction.

Case $(i i)(1)(d)\left(c_{1} \neq 0, c_{2} \neq 0, b=0\right)$. When $1-2 p_{2} \zeta / \eta \neq 0$, we have similar discussions. When $1-2 p_{2} \zeta / \eta=0$, we have $(\lambda+$ 
2) $\eta / \zeta^{2}=1$. By $b=0$, then $\lambda=0$ and $2 \eta=\zeta^{2}=4 p_{2} \zeta$, so $4 p_{2}=\zeta$ and $p_{1}=2 p_{2}$. But $\eta=2 p_{2} \zeta$; then $p_{1}=p_{2}=0$. This is a contradiction.

Case (ii)(2) $\left(\lambda=\zeta^{2} / 4 \eta, \psi=c_{1} e^{(1 / 2) t}+c_{2} t e^{(1 / 2) t}\right)$. By (31), we have

$$
\begin{aligned}
& {\left[\frac{1}{2} c_{1}+c_{2}-a_{0} c_{1}+\left(\frac{c_{2}}{2}-a_{0} c_{2}\right) t\right] e^{(1 / 2) t}} \\
& \quad=\frac{p_{1} \lambda_{2} \eta}{\left(p_{2}-p_{1}\right) \zeta^{2}}\left(c_{1}+c_{2} t\right)^{1-2 p_{2} \zeta / \eta}\left(e^{(1 / 2) t}\right)^{1-2 p_{2} \zeta / \eta},
\end{aligned}
$$

where $a_{0}=1 / 4+2 \eta / \zeta^{2}$.

Case (ii)(2)(a) $\left(c_{2} \neq 0\right)$. One has $(1 / 2) c_{1}+c_{2}-a_{0} c_{1}+\left(c_{2} / 2-\right.$ $\left.a_{0} c_{2}\right) t \neq 0$ and $p_{2}=0$. By (30c), $\lambda_{2}+\left(\phi^{\zeta}\right)^{\prime} / \phi^{\zeta}=\lambda+2$, then $\phi^{\zeta}=c_{0} e^{\left(-\lambda_{2}+\lambda+2\right) t}$ and $\left(c_{1} e^{(1 / 2) t}+c_{2} t e^{(1 / 2) t}\right)^{\zeta^{2} / \eta}=c_{0} e^{\left(-\lambda_{2}+\lambda+2\right) t}$; this is a contradiction with $c_{2} \neq 0$.

Case (ii)(2)(b) ( $\left.c_{2}=0\right)$. By (37), we have

$$
\begin{aligned}
& \left(\frac{1}{2} c_{1}-a_{0} c_{1}\right) e^{(1 / 2) t} \\
& \quad=\frac{p_{1} \lambda_{2} \eta}{\left(p_{2}-p_{1}\right) \zeta^{2}}\left(c_{1}\right)^{1-2 p_{2} \zeta / \eta}\left(e^{(1 / 2) t}\right)^{1-2 p_{2} \zeta / \eta}
\end{aligned}
$$

If $a_{0}=1 / 2$, then $p_{1} \lambda_{2}=0$ and $\lambda=2$. If $p_{1}=0$, then $\zeta=2 p_{2}$ and by (30b), $\left(\phi^{\zeta}\right)^{\prime} / \phi^{\zeta}=4$ and $\phi^{\zeta}=c_{0} e^{4 t}$. By (30c),

$$
\frac{\lambda_{2}}{\left(c_{0}^{\prime} e^{(1 / 2) t}\right)^{2 p_{2} \zeta / \eta}}-\frac{16 p_{2}}{\zeta}+4 \frac{\zeta+2 p_{2}}{\zeta}=4
$$

so $\lambda_{2}=0$ and we have a contradiction by (39).

If $p_{1} \neq 0$, then $\lambda_{2}=0$, so $\psi=c_{1} e^{(1 / 2) t}$ and $\phi^{\zeta}=c_{0} e^{4 t}$. Then by (30b), we have $p_{1}=0$ which contradicts with $p_{1} \neq 0$.

If $a_{0} \neq 1 / 2$, then $p_{2}=0$ and $\zeta=p_{1}, \eta=p_{1}^{2}$ and $\lambda=$ $1 / 4$ and $a_{0}=9 / 4$. By $\phi^{\xi}=c_{1} e^{(1 / 2) t}$ and (30b), we have a contradiction. In a word, we have no solutions in case (ii)(2).

Case (ii)(3). One has $\lambda>\zeta^{2} / 4 \eta, \psi=c_{1} e^{(1 / 2) t} \cos (a t)+$ $c_{2} e^{(1 / 2) t} \sin (a t)$, where $a=\sqrt{4 \lambda \eta / \zeta^{2}-1} / 2$. By (31), we have

$$
\begin{aligned}
\left(\frac{c_{1}}{2}+a c_{2}\right) \cos (a t)+\left(-a c_{1}+\frac{c_{2}}{2}\right) \sin (a t) \\
=\frac{p_{1} \lambda_{2} \eta}{\left(p_{2}-p_{1}\right) \zeta^{2}}\left(c_{1} \cos (a t)+c_{2} \sin (a t)\right)^{1-2 p_{2} \zeta / \eta} e^{-2 p_{2} \zeta / \eta} \\
\quad+\frac{(\lambda+2) \eta}{\zeta^{2}}\left(c_{1} \cos (a t)+c_{2} \sin (a t)\right) .
\end{aligned}
$$

If $p_{2} \neq 0$, then $p_{1} \lambda_{2}=0$ and

$$
\frac{c_{1}}{2}+a c_{2}=\frac{(\lambda+2) \eta}{\zeta^{2}} c_{1}, \quad-a c_{1}+\frac{c_{2}}{2}=\frac{(\lambda+2) \eta}{\zeta^{2}} c_{2},
$$

so $c_{1}^{2}+c_{2}^{2}=0$. This is a contradiction.

If $p_{2}=0$, then

$$
\begin{gathered}
\frac{c_{1}}{2}+a c_{2}=\frac{p_{1} \lambda_{2} \eta}{\left(p_{2}-p_{1}\right) \zeta^{2}} c_{1}+\frac{(\lambda+2) \eta}{\zeta^{2}} c_{1}, \\
-a c_{1}+\frac{c_{2}}{2}=\frac{p_{1} \lambda_{2} \eta}{\left(p_{2}-p_{1}\right) \zeta^{2}} c_{2}+\frac{(\lambda+2) \eta}{\zeta^{2}} c_{2} .
\end{gathered}
$$

Then $c_{1}^{2}+c_{2}^{2}=0$. This is a contradiction. By the above discussions, we get the following theorem.

Theorem 35. Let $M=I \times_{\phi^{p_{1}}} F_{1} \times_{\phi^{p_{2}}} F_{2}$ be a generalized Kasner space-time and $\operatorname{dim} F_{1}=1, \operatorname{dim} F_{2}=2$ and $P=\partial / \partial t$. Then $(M, \bar{\nabla})$ is Einstein with the Einstein constant $\lambda$ if and only if $\left(F_{2}, \nabla^{F_{2}}\right)$ is Einstein with the Einstein constant $\lambda_{2}$, and one of the following conditions is satisfied:

(1) $p_{2}=0, p_{1} \neq 0, \phi=c_{0} e^{t / p_{1}}, \lambda=0, \lambda_{2}=1$;

(2) $p_{1}=0, p_{2} \neq 0, \lambda=0, \lambda_{2}=0, \phi=c_{0} e^{t / p_{2}}$;

(3) $p_{1} \neq 0, p_{2} \neq 0, \lambda=\lambda_{2}=0, p_{1}=4 p_{2}, \phi=c_{0} e^{t / 3 p_{2}}$.

5.2. Type (II) Generalized Kasner Space-Times with a Semisymmetric Metric Connection with Constant Scalar Curvature. By Proposition 33, then $\left(F_{2}, \nabla^{F_{2}}\right)$ has constant scalar curvature $S^{F_{2}}$ and

$$
\bar{S}=\frac{S^{F_{2}}}{\phi^{2} p_{2}}-2 \zeta \frac{\phi^{\prime \prime}}{\phi}-\left(\eta+\zeta^{2}-2 \zeta\right) \frac{\phi^{\prime 2}}{\phi^{2}}+6 \zeta \frac{\phi^{\prime}}{\phi}-6 .
$$

If $\zeta=0$, when $\eta=0$, then $p_{1}=p_{2}=0$ and $\bar{S}=S^{F_{2}}-6$. If $\eta \neq 0$, then

$$
\eta \frac{\phi^{\prime 2}}{\phi^{2}}=\frac{S^{F_{2}}}{\phi^{2} p_{2}}-(\bar{S}+6)
$$

If $\zeta \neq 0$, putting $\phi=\psi^{2 \zeta /\left(\eta+\zeta^{2}\right)}$, we get

$$
-\frac{4 \zeta^{2}}{\eta+\zeta^{2}} \psi^{\prime \prime}+\frac{12 \zeta^{2}}{\eta+\zeta^{2}} \psi^{\prime}-(\bar{S}+6) \psi+S^{F_{2}} \psi^{1-4 p_{2} \zeta /\left(\eta+\zeta^{2}\right)}=0 .
$$

5.3. Type (III) Generalized Kasner Space-Times with a Semisymmetric Metric Connection with Constant Scalar Curvature. By Proposition 33, then

$$
\bar{S}=-2 \zeta \frac{\phi^{\prime \prime}}{\phi}-\left(\eta+\zeta^{2}-2 \zeta\right) \frac{\phi^{\prime 2}}{\phi^{2}}+6 \zeta \frac{\phi^{\prime}}{\phi}-6 .
$$

If $\zeta=\eta=0$, then $p_{1}=p_{2}=p_{3}=0$, and we get $\bar{S}=-6$.

If $\zeta=0, \eta \neq 0$, then $\left[(\ln \phi)^{\prime}\right]^{2}=-(\bar{S}+6) / \eta$, so when $\bar{S}+6>$ 0 , there are no solutions, when $\bar{S}+6=0, \phi$ is a constant, and when $\bar{S}+6<0, \phi=c_{0} e^{ \pm(\sqrt{-(\bar{S}+6) / \eta}) t}$. 
If $\zeta \neq 0$, then $\eta \neq 0$; putting $\phi=\psi^{2 \zeta /\left(\eta+\zeta^{2}\right)}$, then

$$
\psi^{\prime \prime}-3 \psi^{\prime}+\frac{(\bar{S}+6)\left(\eta+\zeta^{2}\right)}{4 \zeta^{2}} \psi=0 .
$$

So, we get

(1) $\bar{S}+6<9 \zeta^{2} /\left(\eta+\zeta^{2}\right), \psi=c_{1} e^{\left(\left(3+\sqrt{9-(\bar{S}+6)\left(\eta+\zeta^{2}\right) / \zeta^{2}}\right) / 2\right) t}+$ $c_{2} e^{\left(\left(3-\sqrt{9-(\bar{S}+6)\left(\eta+\zeta^{2}\right) / \zeta^{2}}\right) / 2\right) t}$

(2) $\bar{S}+6=9 \zeta^{2} /\left(\eta+\zeta^{2}\right), \psi=c_{1} e^{(3 / 2) t}+c_{2} t e^{(3 / 2) t}$,

(3) $\bar{S}+6>9 \zeta^{2} /\left(\eta+\zeta^{2}\right), \psi=$ $c_{1} e^{(3 / 2) t} \cos \left(\left(\sqrt{-9+(\bar{S}+6)\left(\eta+\zeta^{2}\right) / \zeta^{2}} / 2\right) t\right)$ $c_{2} e^{(3 / 2) t} \sin \left(\left(\sqrt{-9+(\bar{S}+6)\left(\eta+\zeta^{2}\right) / \zeta^{2}} / 2\right) t\right)$. we get the following theorem.

Theorem 36. Let $M=I \times_{\phi^{p_{1}}} F_{1} \times_{\phi^{p_{2}}} F_{2} \times_{\phi^{p_{3}}} F_{3}$ be a generalized Kasner space-time and $\operatorname{dim} F_{1}=\operatorname{dim} F_{2}=\operatorname{dim} F_{3}=1$, and $P=\partial / \partial t$. Then $\bar{S}$ is a constant if and only if one of the following cases holds.

(1) One has $\zeta=\eta=0, \bar{S}=-6$.

(2) One has $\zeta=0, \eta \neq 0$; when $\bar{S}+6>0$, there are no solutions, when $\bar{S}+6=0, \phi$ is a constant, and when $\bar{S}+6<0, \phi=c_{0} e^{ \pm \sqrt{-((\bar{S}+6) / \eta) t}}$.

(3) If $\zeta \neq 0$

$$
\begin{aligned}
& \text { (a) } \bar{S}+6 \quad+6 \zeta^{2} /\left(\eta+\zeta^{2}\right), \phi= \\
&\left(c_{1} e^{\left(3+\sqrt{9-(\bar{S}+6)\left(\eta+\zeta^{2}\right) / \zeta^{2}} / 2\right) t}\right. \\
&\left.c_{2} e^{\left(3-\sqrt{9-(\bar{S}+6)\left(\eta+\zeta^{2}\right) / \zeta^{2}} / 2\right) t}\right)^{2 \zeta /\left(\eta+\zeta^{2}\right)}, \\
& \text { (b) } \bar{S}+6=9 \zeta^{2} /\left(\eta+\zeta^{2}\right), \quad \phi= \\
& \\
&\left(c_{1} e^{(3 / 2) t}+c_{2} t e^{(3 / 2) t}\right)^{2 \zeta /\left(\eta+\zeta^{2}\right)}, \\
& \text { (c) } \bar{S}+6>9 \zeta^{2} /\left(\eta+\zeta^{2}\right), \phi=\left(c_{1} e^{(3 / 2) t}\right. \\
& \cos \left(\left(\sqrt{-9+(\bar{S}+6)\left(\eta+\zeta^{2}\right) / \zeta^{2}} / 2\right) t\right)+c_{2} e^{(3 / 2) t} \\
&\left.\sin \left(\left(\sqrt{-9+(\bar{S}+6)\left(\eta+\zeta^{2}\right) / \zeta^{2}} / 2\right) t\right)\right)^{2 \zeta /\left(\eta+\zeta^{2}\right)} .
\end{aligned}
$$

5.4. Einstein Type (III) Generalized Kasner Space-Times with a Semisymmetric Metric Connection. By Proposition 32, we have

$$
\begin{gathered}
\zeta\left(\frac{\phi^{\prime}-\phi^{\prime \prime}}{\phi}\right)-(\eta-\zeta) \frac{\phi^{\prime 2}}{\phi^{2}}=\lambda, \\
-p_{1}\left[\frac{\phi^{\prime \prime}}{\phi}+(\zeta-1) \frac{\phi^{\prime 2}}{\phi^{2}}-2 \frac{\phi^{\prime}}{\phi}\right]+\zeta \frac{\phi^{\prime}}{\phi}=\lambda+2, \\
-p_{2}\left[\frac{\phi^{\prime \prime}}{\phi}+(\zeta-1) \frac{\phi^{\prime 2}}{\phi^{2}}-2 \frac{\phi^{\prime}}{\phi}\right]+\zeta \frac{\phi^{\prime}}{\phi}=\lambda+2, \\
-p_{3}\left[\frac{\phi^{\prime \prime}}{\phi}+(\zeta-1) \frac{\phi^{\prime 2}}{\phi^{2}}-2 \frac{\phi^{\prime}}{\phi}\right]+\zeta \frac{\phi^{\prime}}{\phi}=\lambda+2 .
\end{gathered}
$$

If $\zeta=\eta=0$, by (48a), $\lambda=0$ and by (48b), $\lambda=-2$; this is a contradiction.

If $\zeta=0, \eta \neq 0$, adding (48b), (48c), and (48d), we get $\lambda=-2$. By (48a), $\phi^{\prime 2} / \phi^{2}=2 / \eta$ and $\phi=c_{0} e^{ \pm \sqrt{2 / \eta} t}$. But by (48b), then $\phi^{\prime \prime} / \phi+(\zeta-1)\left(\phi^{\prime 2} / \phi^{2}\right)-2 \phi^{\prime} / \phi=0$, and this is a contradiction.

Consider $\zeta \neq 0$. If $p_{1}=p_{2}=p_{3}$, we get type (I), so we may let $p_{1} \neq p_{2}$. By (48b) and (48c), we have $\left(\phi^{\zeta}\right)^{\prime} / \phi^{\zeta}=\lambda+2$ and $\left(\phi^{\zeta}\right)^{\prime \prime} / \phi^{\zeta}-2\left(\phi^{\zeta}\right)^{\prime} / \phi^{\zeta}=0$, so $\phi^{\zeta}=c_{0} e^{(\lambda+2) t}$ and $\lambda=-2$ or 0 . When $\lambda=-2, \psi$ is a constant; by (48a), $\lambda=0$, this is a contradiction. When $\lambda=0, \psi=c_{0} e^{2 \eta t / \zeta^{2}}$, and $\psi^{\prime \prime}-\psi^{\prime}=0$, so $2 \eta / \zeta^{2}=1$. In this case, we get when $p_{i} \neq p_{j}$ for some $i, j \in$ $\{1,2,3\}, \lambda=0,2 \eta / \zeta^{2}=1, \phi=c_{0} e^{2 t / \zeta}$. We get the following theorem.

Theorem 37. Let $M=I \times_{\phi^{p_{1}}} F_{1} \times_{\phi^{p_{2}}} F_{2} \times_{\phi^{p_{3}}} F_{3}$ be a generalized Kasner space-time for $p_{i} \neq p_{j}$ for some $i, j \in\{1,2,3\}$ and $\operatorname{dim} F_{1}=\operatorname{dim} F_{2}=\operatorname{dim} F_{3}=1$, and $P=\partial / \partial t$. Then $(M, \bar{\nabla})$ is Einstein with the Einstein constant $\lambda$ if and only if $\lambda=0,2 \eta / \zeta^{2}=1, \phi=c_{0} e^{2 t / \zeta}$.

Remark 38. Comparing with Proposition 4.3, Proposition 4.11, Section 5 in [5], in Proposition 32, Proposition 33, and Theorems 35-37, the unit vector field $P=\partial / \partial t$ emerges. For Proposition 4.3, Proposition 4.11, Section 5 in [5], $P$ equals zero. So equations in our theorems are different from the equations in theorems in [5].

\section{Conflict of Interests}

The author declares that there is no conflict of interests regarding the publication of this paper.

\section{Acknowledgments}

This work was supported by NCET-13-0721 and NSFC 11271062. The author would like to thank referees for their careful reading and helpful comments.

\section{References}

[1] R. L. Bishop and B. O’Neill, "Manifolds of negative curvature," Transactions of the American Mathematical Society, vol. 145, pp. $1-49,1969$.

[2] F. Dobarro and E. L. Dozo, "Scalar curvature and warped products of Riemann manifolds," Transactions of the American Mathematical Society, vol. 303, no. 1, pp. 161-168, 1987.

[3] P. E. Ehrlich, Y.-T. Jung, and S.-B. Kim, "Constant scalar curvatures on warped product manifolds," Tsukuba Journal of Mathematics, vol. 20, no. 1, pp. 239-256, 1996.

[4] L. J. Alías, A. Romero, and M. Sánchez, "Spacelike hypersurfaces of constant mean curvature and Calabi-Bernstein type problems," The Tohoku Mathematical Journal, vol. 49, no. 3, pp. 337$345,1997$.

[5] F. Dobarro and B. Ünal, "Curvature of multiply warped products," Journal of Geometry and Physics, vol. 55, no. 1, pp. 75-106, 2005. 
[6] M. Fernández-López, E. García-Río, D. N. Kupeli, and B. Ünal, "A curvature condition for a twisted product to be a warped product," Manuscripta Mathematica, vol. 106, no. 2, pp. 213-217, 2001.

[7] H. A. Hayden, "Sub-spaces of a space with torsion," Proceedings of the London Mathematical Society, vol. 34, no. 1, pp. 27-50, 1932.

[8] K. Yano, "On semi-symmetric metric connection," Revue Roumaine de Mathématiques Pures et Appliquées, vol. 15, pp. 1579-1586, 1970.

[9] S. Sular and C. Özgür, "Warped products with a semisymmetric metric connection," Taiwanese Journal of Mathematics, vol. 15, no. 4, pp. 1701-1719, 2011.

[10] F. Pfäffle and C. A. Stephan, “The Holst action by the spectral action principle," Communications in Mathematical Physics, vol. 307, no. 1, pp. 261-273, 2011.

[11] F. Pfäffle and C. A. Stephan, "Chiral asymmetry and the spectral action," Communications in Mathematical Physics, vol. 321, no. 2, pp. 283-310, 2013.

[12] F. W. Hehl, P. von der Heyde, G. D. Kerlick, and J. M. Nester, "General relativity with spin and torsion: foundations and prospect," Reviews of Modern Physics, vol. 48, no. 3, pp. 393-416, 1976.

[13] I. L. Shapiro, "Physical aspects of the space-time torsion," Physics Reports, vol. 357, no. 2, pp. 113-213, 2002. 


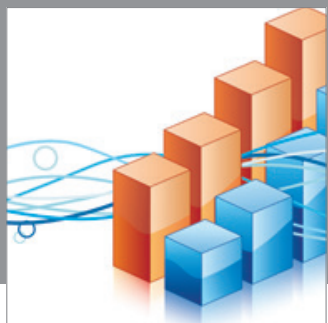

Advances in

Operations Research

mansans

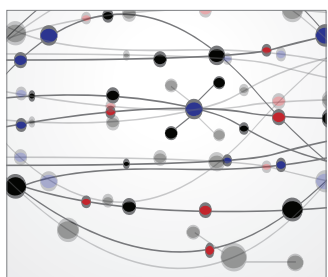

The Scientific World Journal
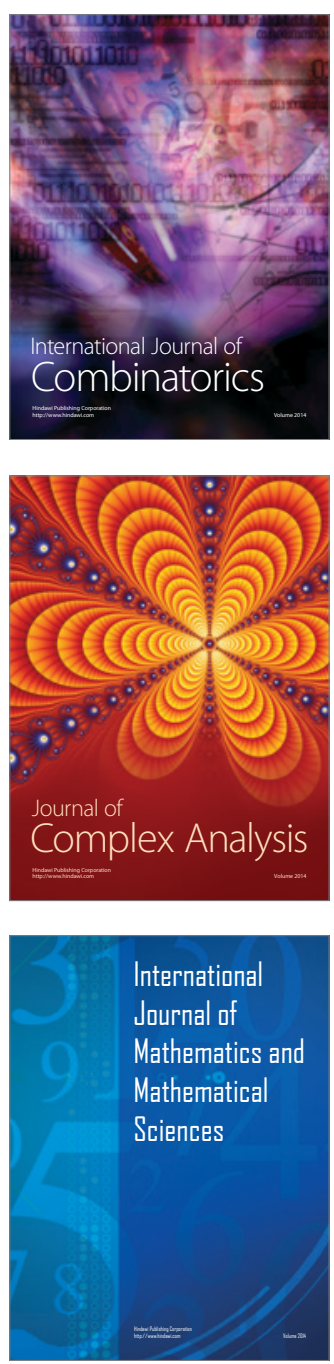
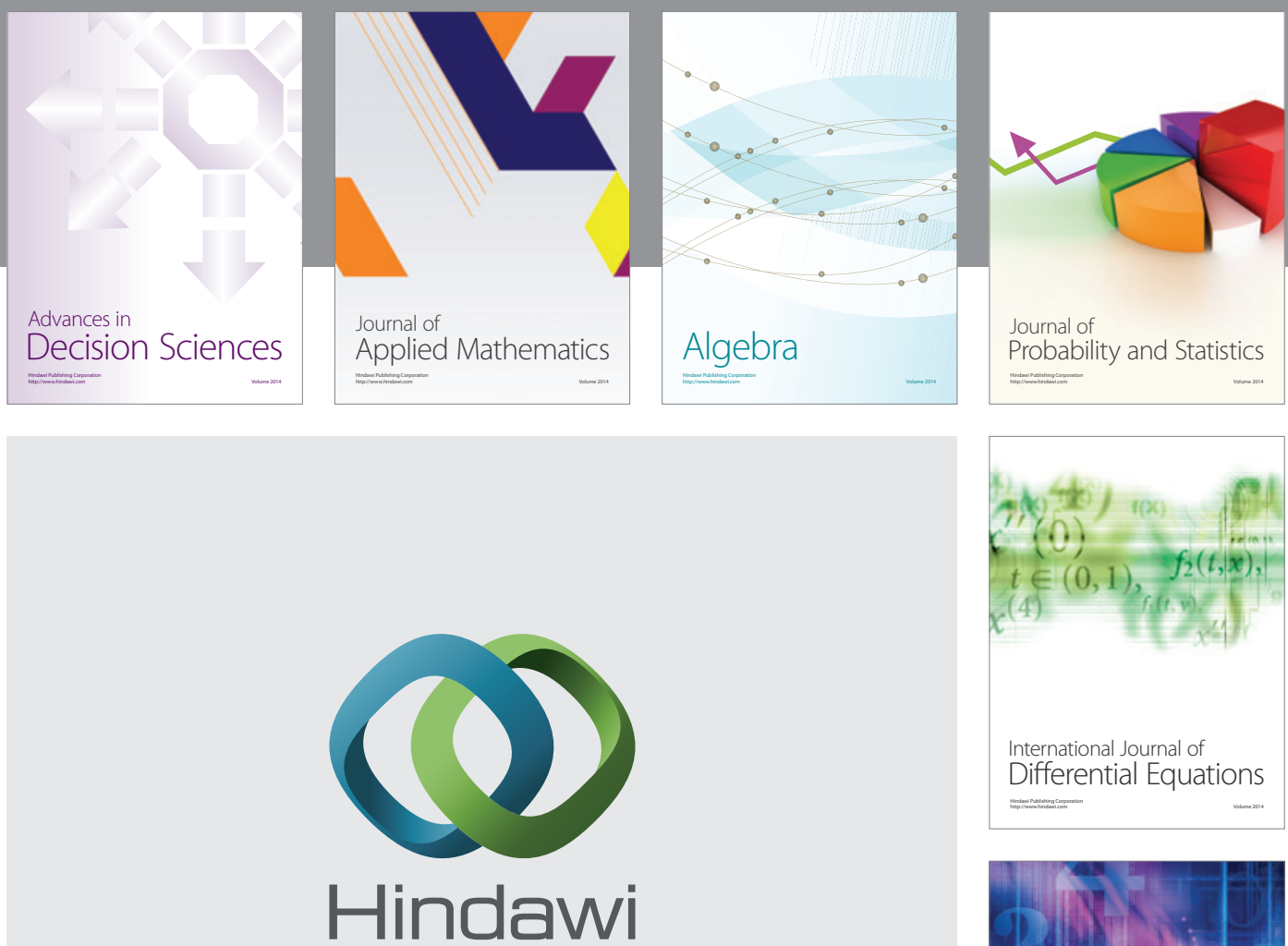

Submit your manuscripts at http://www.hindawi.com
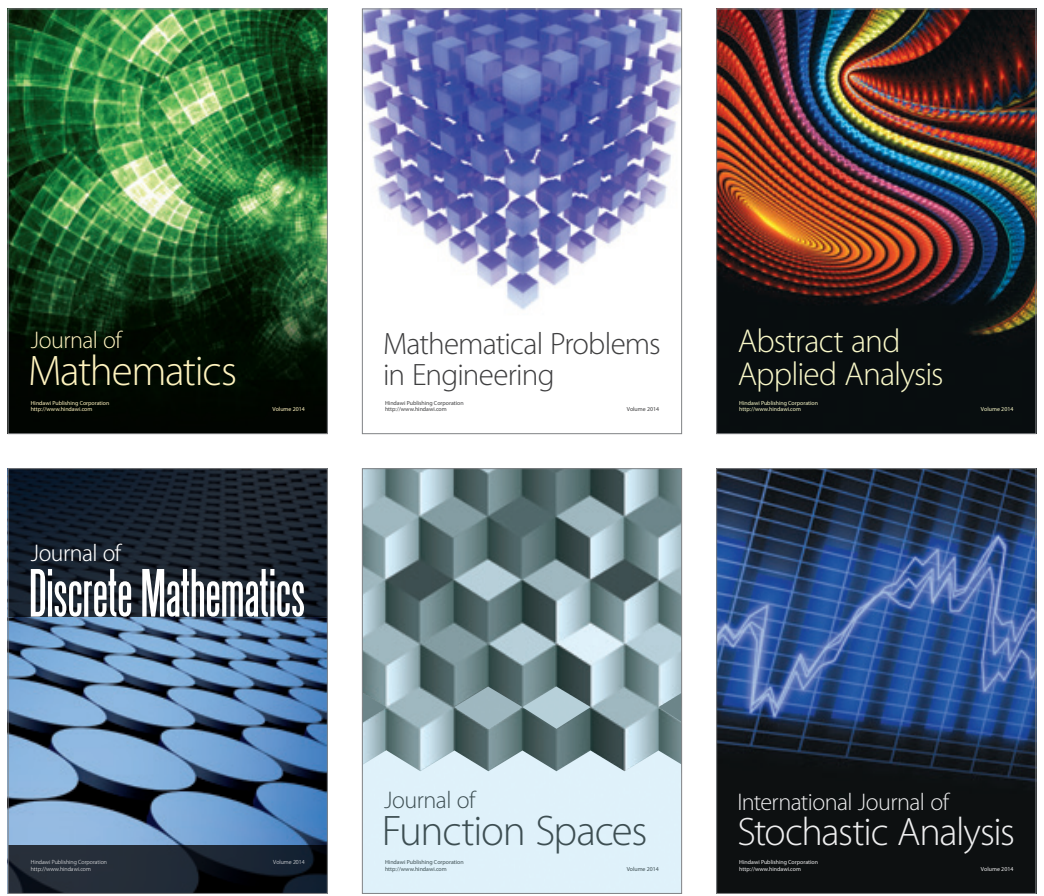

Journal of

Function Spaces

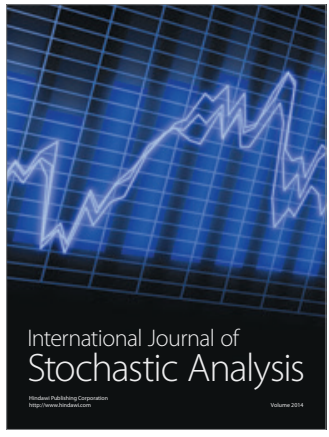

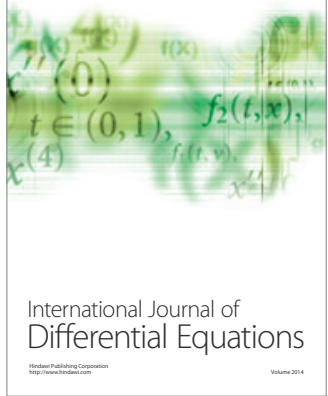
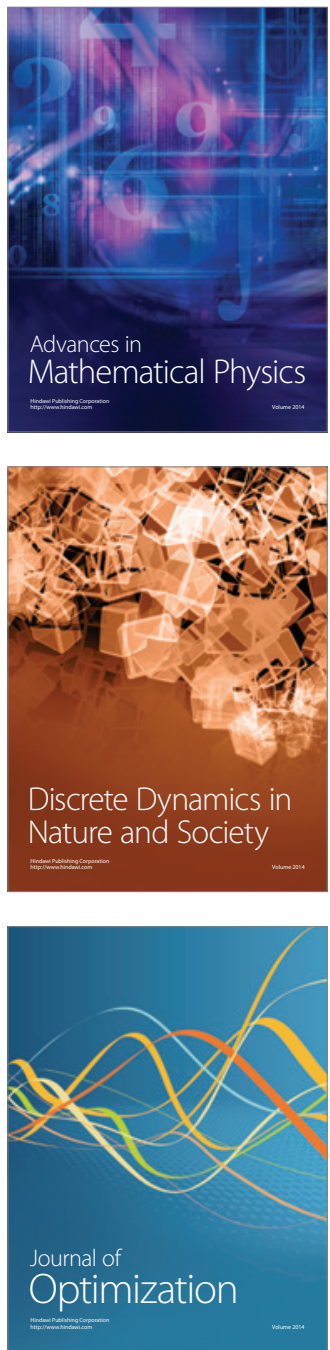